\title{
SPICAV on Venus Express: Three spectrometers to study the global structure and composition of the Venus atmosphere
}

Jean-Loup Bertaux ${ }^{\mathrm{a}, *}$, D. Nevejans ${ }^{\mathrm{b}}$, O. Korablev ${ }^{\mathrm{c}}$, E. Villard $^{\mathrm{a}}$, E. Quémerais ${ }^{\mathrm{a}}$, E. Neefs $^{\mathrm{b}}$, F. Montmessin ${ }^{\mathrm{a}}$, F. Leblanc ${ }^{\mathrm{a}}$, J.P. Dubois ${ }^{\mathrm{a}}$, E. Dimarellis ${ }^{\mathrm{a}}$, A. Hauchecorne ${ }^{\mathrm{a}}$, F. Lefèvre ${ }^{\mathrm{a}}$, P. Rannou ${ }^{a}$, J.Y. Chaufray ${ }^{\text {a }}$, M. Cabane ${ }^{\text {a }}$, G. Cernogora ${ }^{a}$, G. Souchon ${ }^{a}$, F. Semelin ${ }^{\text {, }}$, A. Reberac ${ }^{\mathrm{a}}$, E. Van Ransbeek ${ }^{\mathrm{b}}$, S. Berkenbosch ${ }^{\mathrm{b}}$, R. Clairquin ${ }^{\mathrm{b}}$, C. Muller ${ }^{\mathrm{b}}$, F. Forget $^{\mathrm{d}}$,

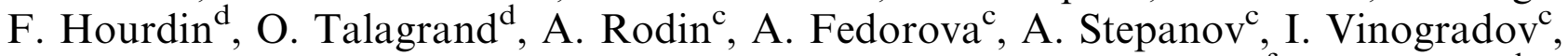
A. Kiselev' ${ }^{\text {c }}$ Yu. Kalinnikov ${ }^{\text {, }}$ Georges Durry ${ }^{\mathrm{g}}$, B. Sandel ${ }^{\mathrm{e}}$, A. Stern ${ }^{\mathrm{f}}$, J.C. Gérard ${ }^{\mathrm{h}}$

\author{
${ }^{a}$ Service d'Aéronomie du CNRS, 91371, Verrières-le-Buisson, France \\ ${ }^{\mathrm{b}}$ Belgian Institute for Space Aeronomy, 3 av. Circulaire, B-1180 Brussels, Belgium \\ ${ }^{\mathrm{c}}$ Space Research Institute (IKI), 84/32 Profsoyuznaya, 117810 Moscow, Russia \\ ${ }^{\mathrm{d}}$ Laboratoire de Météorologie Dynamique, 4 place Jussieu, 75252 Paris Cedex 05, Paris, France \\ ${ }^{\mathrm{e}}$ Lunar and Planetary Laboratory, University of Arizona, 1541 E. University Blvd., Tucson, AZ 85721, USA \\ ${ }^{\mathrm{f}}$ Southwest Research Institute, Geophys., Astrophys., and Planet. Sci., 1050 Walnut Ave., Suite 400, Boulder, CO 80302-5143, USA \\ ${ }^{\mathrm{g}}$ Groupe de Spectrométrie Moléculaire et Atmosphérique, Université de Reims Champagne-Ardennes B.P.1039, 51687 Reims Cedex, France \\ ${ }^{\mathrm{h}}$ Université de Liège, Institut d'Astrophysique et Géophysique-B5c, Allée du 6 aout, 17—Sart Tilman, B-4000 Liège, Belgium
}

Accepted 10 April 2006

Available online 9 February 2007

\begin{abstract}
Spectroscopy for the investigation of the characteristics of the atmosphere of Venus (SPICAV) is a suite of three spectrometers in the UV and IR range with a total mass of $13.9 \mathrm{~kg}$ flying on the Venus Express (VEX) orbiter, dedicated to the study of the atmosphere of Venus from ground level to the outermost hydrogen corona at more than $40,000 \mathrm{~km}$. It is derived from the SPICAM instrument already flying on board Mars Express (MEX) with great success, with the addition of a new IR high-resolution spectrometer, solar occultation IR (SOIR), working in the solar occultation mode. The instrument consists of three spectrometers and a simple data processing unit providing the interface of these channels with the spacecraft.

A UV spectrometer $(118-320 \mathrm{~nm}$, resolution $1.5 \mathrm{~nm}$ ) is identical to the MEX version. It is dedicated to nadir viewing, limb viewing and vertical profiling by stellar and solar occultation. In nadir orientation, SPICAV UV will analyse the albedo spectrum (solar light scattered back from the clouds) to retrieve $\mathrm{SO}_{2}$, and the distribution of the UV-blue absorber (of still unknown origin) on the dayside with implications for cloud structure and atmospheric dynamics. On the nightside, $\gamma$ and $\delta$ bands of NO will be studied, as well as emissions produced by electron precipitations. In the stellar occultation mode the UV sensor will measure the vertical profiles of $\mathrm{CO}_{2}$, temperature, $\mathrm{SO}_{2}, \mathrm{SO}$, clouds and aerosols. The density/temperature profiles obtained with SPICAV will constrain and aid in the development of dynamical atmospheric models, from cloud top $(\sim 60 \mathrm{~km})$ to $160 \mathrm{~km}$ in the atmosphere. This is essential for future missions that would rely on aerocapture and aerobraking. UV observations of the upper atmosphere will allow studies of the ionosphere through the emissions of $\mathrm{CO}, \mathrm{CO}^{+}$, and $\mathrm{CO}_{2}^{+}$, and its direct interaction with the solar wind. It will study the $\mathrm{H}$ corona, with its two different scale heights, and it will allow a better understanding of escape mechanisms and estimates of their magnitude, crucial for insight into the long-term evolution of the atmosphere.

The SPICAV VIS-IR sensor $(0.7-1.7 \mu \mathrm{m}$, resolution $0.5-1.2 \mathrm{~nm})$ employs a pioneering technology: an acousto-optical tunable filter (AOTF). On the nightside, it will study the thermal emission peeping through the clouds, complementing the observations of both VIRTIS and Planetary Fourier Spectrometer (PFS) on VEX. In solar occultation mode this channel will study the vertical structure of $\mathrm{H}_{2} \mathrm{O}, \mathrm{CO}_{2}$, and aerosols.
\end{abstract}

\footnotetext{
*Corresponding author. Tel.: + 33169202999 ; fax: + 33164474245 .

E-mail address: bertaux@aerov.jussieu.fr (J.-L. Bertaux).
} 
The SOIR spectrometer is a new solar occultation IR spectrometer in the range $\lambda=2.2-4.3 \mu \mathrm{m}$, with a spectral resolution $\lambda / \Delta \lambda$ $>15,000$, the highest on board VEX. This new concept includes a combination of an echelle grating and an AOTF crystal to sort out one order at a time. The main objective is to measure $\mathrm{HDO}$ and $\mathrm{H}_{2} \mathrm{O}$ in solar occultation, in order to characterize the escape of D atoms from the upper atmosphere and give more insight about the evolution of water on Venus. It will also study isotopes of $\mathrm{CO}_{2}$ and minor species, and provides a sensitive search for new species in the upper atmosphere of Venus. It will attempt to measure also the nightside emission, which would allow a sensitive measurement of HDO in the lower atmosphere, to be compared to the ratio in the upper atmosphere, and possibly discover new minor atmospheric constituents.

(C) 2007 Elsevier Ltd. All rights reserved.

Keywords: Venus; Atmosphere; Aurorae and airglow; Composition; Occultation

\section{Context of Venus Express mission and SPICAV overview}

When ESA decided to issue an AO for a new flexible mission, the SPICAM team on Mars Express (MEX) decided to join the group of scientists who wished to propose a mission to Venus, with a spacecraft as similar as possible to MEX, and instruments already "on the shelf", or additional copies of existing instruments. This was the case for VIRTIS, which is now flying on board Rosetta. Its infrared (IR) capabilities make it the most important instrument of the Venus Express (VEX) payload, because it allows "seeing" through the clouds the thermal emission from the ground and lower atmosphere, which was discovered from Earth-bound telescope observations, glimpsed by Galileo and Cassini during their fly-bys, but never studied from an orbiter around Venus.

In the context of a flexible mission, which has to be of moderate cost and fast development, it was natural for the MEX SPICAM team to propose a reflight of SPICAM. But at the same time, two of us (Korablev and Bertaux) were designing a new type of high-resolution spectrometer; it became obvious that flying such a device to Venus, in the solar occultation mode, would provide unique information about the $\mathrm{HDO}$ and $\mathrm{H}_{2} \mathrm{O}$ content of the upper atmosphere of Venus, crucial for the understanding of the history of water on Venus. Dennis Nevejans and his team at BIRAIASB obtained the necessary funding from the Belgian Federal Science Policy Office to design and manufacture the solar occultation IR (SOIR) spectrometer in collaboration with a Belgian industrial partner (OIP). SOIR became a part of SPICAV.

While SPICAM on MEX is a 4.7-kg UV-IR instrument, we had to stack SOIR on top of SPICAM to produce a single mechanical stand-alone package, that we call SPICAV, with a mass of $13.9 \mathrm{~kg}$. This implied a redesign of the mechanical structure of SPICAM. For VEX, the optical arrangement is the same as for MEX, but the cover of SPICAM is replaced by stiff walls able to support a second floor, where SOIR is mounted.

Therefore, SPICAV is a suite of three different spectrometers. The SPICAV UV spectrometer is refurbished from the spare flight model of SPICAM prepared for the MEX mission. The SPICAV IR channel has been substantially modified so that a completely new qualification model and two flight models have been fabricated for VEX. SOIR is a conceptually new instrument, but in spite of numerous difficulties due to a very tight schedule, the complete protoflight model of SOIR was completed and tested in early 2005 , and finally mounted on the VEX spacecraft in May 2005, with a successful launch on November 9, 2005.

Because during some mission periods the sun faces the optical instruments, we also developed in a very short time two additional hardware items: two optical baffles which are mounted on the $+Z$ face of the spacecraft (the common bore sight axis of all optical instruments), and a mechanical shutter activated by a stepper motor. Completing SPICAV is a common digital processing unit (DPU), which provides the electrical and data interface to the spacecraft for the three channels.

As a precursor of SPICAV UV, the Pioneer Venus UV spectrometer (PVOUVS) has monitored the top of Venus' clouds and mesospheric airglow in the UV range. With SPICAV UV, which registers all wavelengths at once, the $\mathrm{S} / \mathrm{N}$ ratio will be much greater than for the Pioneer Venus UV scanning spectrometer (larger than 100 for each nanometre of spectrum in $1 \mathrm{~s}$ integration time). SPICAV UV will be the first instrument at Venus to implement the solar/stellar occultation technique that has been proven to be very effective in the studies of the atmospheres of Earth, Mars, and outer planets. The star Spica, when occulted by the moon in the 18th century, was carefully observed by astronomers: its light decreased abruptly when occulted by the dark limb of the moon, and it was correctly concluded that there was no atmosphere around the moon, because otherwise, refraction would have produced a progressive dimming of the star. The SPICAM/SPICAV acronyms are a tribute to this early use of stellar occultation.

In the Earth's atmosphere, the occultation technique has been used to measure $\mathrm{O}_{3}$ since the 1970s. In the early attempts only one or two wavelengths were observed at a time, making the identification of the absorber species uncertain. With the advent of multi-pixel detectors, in a multi-spectral occultation the absorbing species can be safely identified due to their spectral signatures. It also offers the potential to discover new, unexpected species in the atmosphere. The method of absorptive occultation spectroscopy is reviewed in Roscoe et al. (1994) and Smith and Hunten (1990). In the field of Earth's stratospheric research, it has become the most advanced method for longterm monitoring of ozone. In the IR the most remarkable results are those of ATMOS/ATLAS experiment on the shuttle, which provided a set of high-resolution IR 
transmission spectra of the terrestrial atmosphere. In the UV-visible, SAGE-3 from NASA uses a full wavelength coverage on the Sun. On board ENVISAT (ESA, 2001) scanning imaging absorption spectrometer for atmospheric cartography (SCIAMACHY) is performing solar occultation and nadir observations, and the global ozone monitoring by occultations of stars (GOMOS) instrument is fully dedicated to the monitoring of ozone and other species by stellar occultations (about 100,000 per year). Therefore, the methodology of SPICAM and SPICAV, which uses stellar occultations, solar occultations, limb measurements and nadir measurements, is in line with the most advanced instrumentation foreseen for the study of the atmosphere of the Earth.

With VEX in orbit around Venus, the three terrestrial planets, Venus, Earth, and Mars, will have one instrument dedicated to stellar occultations in operation, with GOMOS on ENVISAT completing the trio.

SOIR experiment was proposed for VEX as a lightweight high-resolution solar occultation instrument to study the composition and structure of the atmosphere of Venus above clouds. It is based on a new concept of a compact high-resolution system for remote measurements of gaseous composition with a typical resolution of $\sim 0.2 \mathrm{~cm}^{-1}$ (a resolving power of $15,000-20,000$ ) within a mass budget of $6 \mathrm{~kg}$ and no moving parts. The instrument employs the method of solar occultation. The system consists of an echelle spectrometer, combined with an acousto-optic tunable filter (AOTF) for separation of diffraction orders. The principle of the SOIR instrument and the early prototypes were described by Korablev et al. (2002a, 2004). With regards to the studies of planetary

Table 1

\begin{tabular}{ll}
\hline Spectral bands & UV: $0.55 \mathrm{~nm} /$ pix \\
& IR: $0.8 \mathrm{~nm} /$ pix at $1.5 \mu \mathrm{m}$ \\
& SOIR: $0.32 \mathrm{~cm}^{-1}$ at $2.4 \mu \mathrm{m}$ \\
Spectral sampling & UV: $0.55 \mathrm{~nm} /$ pix \\
& IR: $0.8 \mathrm{~nm} / \mathrm{pix}$ at $1.5 \mu \mathrm{m}$ \\
& SOIR: $0.32 \mathrm{~cm}^{-1}$ at $2.4 \mu \mathrm{m} ; 0.15 \mathrm{~cm}^{-1}$ at $4.0 \mu \mathrm{m}$ \\
& DPU + harness $0.865 \mathrm{~kg}$ \\
Mass & SU $13.05 \mathrm{~kg}$ \\
& Total $13.915 \mathrm{~kg}$ \\
& Sun baffles $0.47 \mathrm{~kg}$ \\
& DPU + SU $17.6 \mathrm{~W}, 26.4 \mathrm{~W}, 51.4 \mathrm{~W}$ \\
Power & DPU: $161 \times 142 \times 70 \mathrm{~mm}^{3}$ \\
Volume & SU: $504 \times 400 \times 350 \mathrm{~mm}^{3}$ \\
& $9,34,66 \mathrm{kbit} / \mathrm{s}$ \\
Data rate & Typ. $100-400 \mathrm{Mbits} / \mathrm{day} \mathrm{TBC}$ \\
Data Volume & One On Board Time TC, One Spicav TC \\
Observations & $5-30 \mathrm{mn}$ typ. \\
Duration & Inertial Star (also used for limb viewing) \\
Pointing (orientation) & Inertial Sun \\
& Nadir \\
\hline &
\end{tabular}

atmospheres this approach offers a dramatic (an order of magnitude) increase in resolving power that leads to significant improvement in sensitivity and accuracy. SOIR for VEX is built by Belgian industry (OIP Sensor Systems) under the responsibility of BIRA-IASB funded via PRODEX contract. In the following, we describe with some details the three spectrometers, their modes of operations, the collected data and the scientific questions that are addressed (Table 1).

\section{Investigations in the ultra-violet with the UV spectrometer channel}

\subsection{UV spectrometer description}

A summary of the characteristics of the UV spectrometer is given in Table 2. It is almost identical to SPICAM UV channel on MEX (Bertaux et al., 2000, 2006). For high efficiency in the UV, the UV spectrometer includes only two reflective surfaces (Fig. 1). The light flux is collected by an off-axis parabolic mirror, which reflects the light toward the entrance of the spectrometer. At its focal plane, a mechanical slit system provides two configurations: with no slit at all, for stellar occultations, and with a slit for extended source observations. In this case the slit defines the FOV, and is the entrance slit of the spectrometer. The slit is divided into two parts with different widths allowing two spectral resolutions when observing an extended source. The first part $(50 \mu \mathrm{m})$ gives good resolution with lower flux; the second part $(500 \mu \mathrm{m})$ gives more sensitivity at the expense of a coarser spectral resolution. The slit can be completely retracted, creating a hole corresponding to the total useful field of view of $1^{\circ} \times 3.16^{\circ}$ (unvignetted) and

Table 2

\begin{tabular}{|c|c|}
\hline Primary mirror & $\begin{array}{l}\text { Off-axis parabola } 40 \times 40 \mathrm{~mm}^{2} \\
\text { Coated } \mathrm{MgF}_{2}, f=120 \mathrm{~mm}\end{array}$ \\
\hline Slit & $\begin{array}{l}50 \mu \mathrm{m} \times 4.6 \mathrm{~mm} \\
500 \mu \mathrm{m} \times 2.2 \mathrm{~mm}\end{array}$ \\
\hline FOV & $\begin{array}{l}\text { Of a pixel } 0.7^{\prime} \times 0.7^{\prime} \\
2^{\circ} \times 3.16^{\circ} \text { no slit (stellar occultation) } \\
0.24^{\circ} \times 0.95^{\circ} \text { with double slit }\end{array}$ \\
\hline Spectral range & $118-320 \mathrm{~nm}$ \\
\hline Grating & $\begin{array}{l}\text { Holographic, concave, toroidal coated } \mathrm{MgF}_{2} \\
2901 / \mathrm{mm} \text {, blazed } 170 \mathrm{~nm}\end{array}$ \\
\hline $\begin{array}{l}\text { Spectral sampling per } \\
\text { pixel }\end{array}$ & $0.52 \mathrm{~nm}$; spectral resolution $1.5 \mathrm{~nm}$ \\
\hline $\begin{array}{l}\text { Resolving power } \\
\text { (occultations) }\end{array}$ & 60-200 stellar; small slit \\
\hline $\begin{array}{l}\text { Resolving power } \\
\text { (extended source) }\end{array}$ & $\begin{array}{l}120-300 \text { small slit, } \\
\approx 20 \text { large slit }\end{array}$ \\
\hline Pointing accuracy & $<0.2^{\circ}$ \\
\hline Detector & $\begin{array}{l}\text { CCD Thomson TH7863 TE cooled at } 270 \mathrm{~K} \text {. } \\
\text { Useful } 288 \times 384 \text { pixels, } 23 \times 23 \mu \mathrm{m}\end{array}$ \\
\hline Intensifier & $\begin{array}{l}\text { Hamamatsu } 200 \mathrm{M} \text {, solar blind } \mathrm{CsTe} \\
\text { photocathode, input window } \mathrm{CaF}_{2}+\mathrm{MgF}_{2}\end{array}$ \\
\hline Vertical resolution & $\begin{array}{l}<1 \mathrm{~km} \text { (occultations) } \\
\sim 10 \mathrm{~km}(\operatorname{limb})\end{array}$ \\
\hline
\end{tabular}




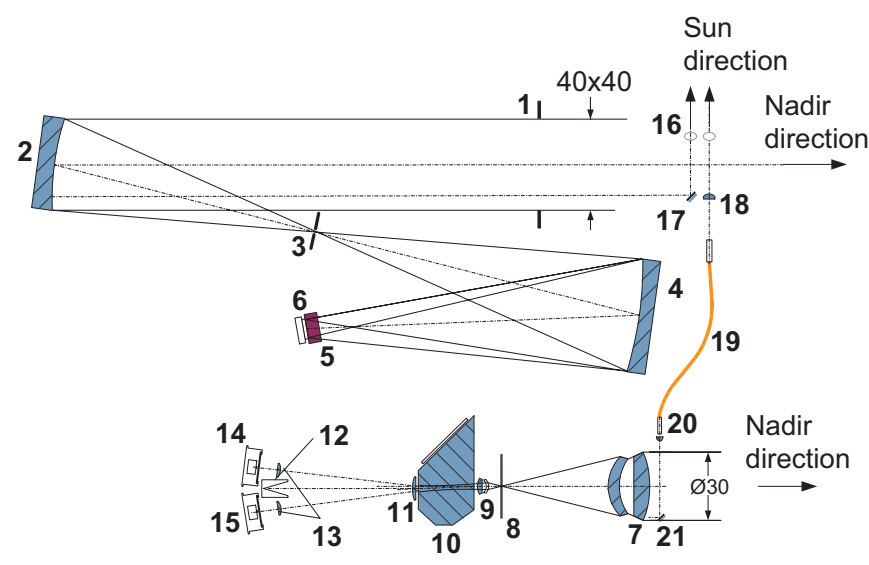

Fig. 1. Optical scheme of the UV and IR channels of SPICAV. 1mechanical stop of the UV channel aperture; 2 - off-axis parabolic mirror; 3 - slit (can be removed by a mechanical actuator, see text); 4 - concave UV grating; 5-intensifier; 6 - CCD; 7-IR channel objective; 8-IR FOV diaphragm; 9, 11- collimating lenses; 10-AOTF crystal; 12-light trap for undiffracted light; 13 - detector proximity lenses; 14 - "extraordinary" beam detector; 15- "ordinary" beam detector; 16 - solar opening (closed by shutter when not looking toward the Sun); 17, 21 - flat mirror; 18-IR solar entry; 19 - optical fibre; 20 - fibre collimator.

$4^{\circ} \times 3.16^{\circ}$ (with vignetting). This configuration is used in the stellar occultation mode at the dark limb when the spectrum of the star is recorded on a few lines of the CCD. The required pointing accuracy is $0.2^{\circ}$, but it is so much better on MEX that it has been possible in some occasions to perform stellar occultations within the narrow part of the slit (only $0.02^{\circ}$ wide).

A holographic concave toroidal grating from JobinYvon, ion-etched for better efficiency feeds the detection block, with the dispersion direction perpendicular to the slit. Therefore, each point of the slit (or the focal plane, when there is no slit) has its spectrum formed perpendicular to the slit, on the photo-cathode of an image intensifier (Hamamatsu) with a CsTe cathode (UV sensitive, but blind to visible light and $\lambda>320 \mathrm{~nm}$ ), microchannel plate electron multiplier, and a phosphor output screen. The image ratio is $\sim 1$, which means that a monochromatic image in the entrance of the spectrometer is conserved in the plane of the detector. The green image from the phosphor is transferred by custom-made fibre optic coupling to a Thomson CCD (TH7863) with $288 \times 384$ useful pixels and a masked zone of equivalent size. Pixel size is $23 \mu \mathrm{m} \times 23 \mu \mathrm{m}$. The 288 lines of the CCD are oriented along the spectral direction, and each line records the spectrum of one point of the entrance slit, with 384 spectral elements sensitive to light and 16 masked pixels, allowing a good measurement of the Dark Charge.

The spectral resolution for a point source determined by aberrations is about $1.5 \mathrm{~nm}$, and 1 pixel of CCD samples $0.54 \mathrm{~nm}$. The CCD detector may be cooled by a Peltier cooler to decrease its temperature and associated dark current. By means of custom-made fibre optics the CCD is coupled with the output window of the image intensifier (from Hamamatsu, type $200 \mathrm{M}$ ). The solar blind CsTe photo-cathode of Hamamatsu intensifier has zero quantum efficiency longward of $320 \mathrm{~nm}$, and is deposited on the internal face of the input window, made of $\mathrm{MgF}_{2}$ to reach down to Lyman $\alpha(\mathrm{L} \alpha)$ wavelength (another target of SPICAV instrumentation). On SPICAM/MEX an additional $\mathrm{CaF}_{2}$ filter was glued on the front surface of the window and covers it in part, preventing overlapping of diffraction orders and $\mathrm{L} \alpha$ stray light. However, the analysis of MEX results inclined us to remove this extra window for SPICAV/VEX, in part because the contribution of the second order in a holographic grating is very small.

The focal length of the telescope is such that one CCD pixel covers a FOV of $0.01 \times 0.01^{\circ}$. The slit of the spectrometer has two parts: a narrow part $\left(0.02^{\circ}\right.$ wide by $1.9^{\circ}$ long), achieving a spectral resolution of $1.5 \mathrm{~nm}$ (about 3 CCD pixels $)$, and a wide part $\left(0.2^{\circ}\right.$ wide by $0.98^{\circ}$ long $)$, achieving a higher photometric sensitivity for extended sources (factor $\sim 8$ ), at the expense of a reduced spectral resolution $(6 \mathrm{~nm})$. In principle SPICAV can record 288 spatially resolved spectra along its $2.88^{\circ}$ long slit (i.e. each spatial element subtending $0.02^{\circ} \times 0.01^{\circ}$ on the sky). However, in practice, only 5 spectra are transmitted for each $1 \mathrm{~s}$ measurement to reduce the volume of the transmitted data. These are usually a sum of $N$ individual CCD line spectra, with $N=1,2,4,8,16$, or 32 , forming 5 adjacent spatial bins of variable extent (from $0.01^{\circ}$ to $\left.0.32^{\circ}\right)$. A sketch of this detector is found in Bertaux et al. (2006).

The gain of the micro-channel plate (MCP) of the image intensifier may be adjusted by telecommand with a high voltage level from 500 to $900 \mathrm{~V}$, commanded by a digital level HT from 0 to 255. A photo-electron created in the photo-cathode (a photo-event), results in a pulse of light from the phosphor, distributed over a few pixels of the $\mathrm{CCD}$. It is detected by the CCD reading electronics as a number of analogue to digital units (ADUs). At HT $=20$ (a usual low gain level necessary to avoid saturation for dayside nadir observations), there are about 2 ADU created per photo-event, while for $\mathrm{HT}=200$, there 40 ADU per photo-event. The absolute calibration of the instrument is well characterized by numerous observations of hot UV stars and comparison with previous International Ultraviolet Explorer (IUE) measurements.

There is a potential danger to activate the intensifier at a large gain on an intense source of light. Therefore, this high gain has to be used cautiously, on the dayside but at high altitude, and then on the nightside. One constraint of SPICAV operation is that during one observing period (ON-OFF), the parameters of the instrument may not be changed. In particular, when a fixed inertial attitude of the spacecraft is chosen, then one must be sure that the signal will not be too strong to damage the intensified detector, if one uses a high gain on a bright target.

\subsubsection{Instrumental characteristics measured on ground}

SPICAV UV underwent a series of laboratory tests, some in full air, others in vacuum (wavelengths below 
a

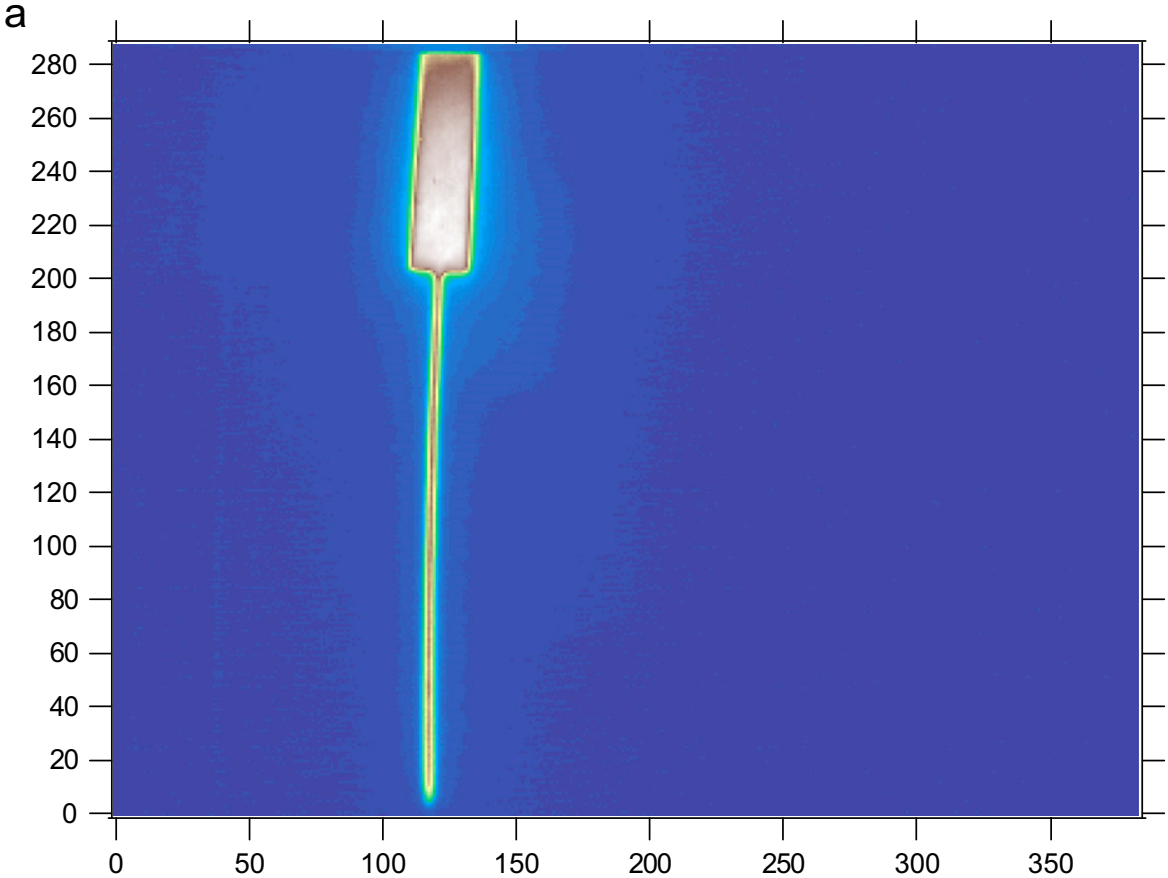

b

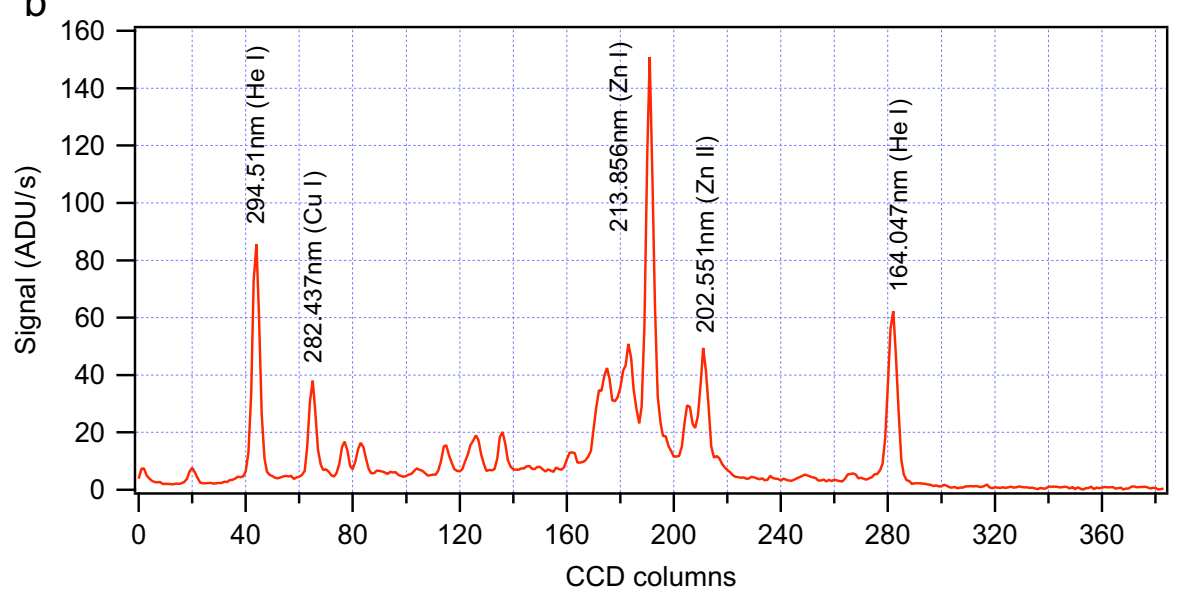

Fig. 2. (a) Full image of the detector when illuminated by a monochromatic light ( $\mathrm{Hg}$ line emission at $253.7 \mathrm{~nm}$ ). The two parts of the slit are well seen, one being larger than the other. It can be noted also some curvature of the slit, due to some image deformation by the optics, indicating a slight change in the wavelength calibration for each line of the CCD. There is also some stray light contamination, mainly coming from the large portion of the slit, which is minimal in the lower portion of the detector. (b) The recorded spectrum of a $\mathrm{HCl}$ discharge lamp seen in vacuum by SPICAV UV channel allows a wavelength calibration of the spectrometer. The wavelength extends from $110 \mathrm{~nm}$ at the right end to $310 \mathrm{~nm}$ at the left end. There is no sign of second order contamination $(\lambda<165 \mathrm{~nm})$ in the first-order spectrum $(\lambda>220 \mathrm{~nm})$.

$190 \mathrm{~nm}$ are absorbed by $\mathrm{O}_{2}$ and $\mathrm{H}_{2} \mathrm{O}$ ). Fig. 2a is a full image of the detector when illuminated by a monochromatic light ( $\mathrm{Hg}$ line emission at $253.7 \mathrm{~nm}$ ). We obtain the image of the slit, with the two parts, one being wider than the other. The figure shows some curvature of the slit, due to image deformation by the optics. Therefore, each line of the CCD must be calibrated in wavelength. This is done (Fig. 2b) by illuminating in vacuum the whole slit with a discharge lamp providing a number of known lines. In addition, numerous tests were performed in order to determine the overall sensitivity. The wavelength reflectivity of each component was tested under vacuum. The detector was calibrated using the method of photon transfer, which yields the number of ADU generated in the CCD by a single photo-electron event, as a function of the high voltage applied to the MCP. The overall sensitivity in flight is determined by observing hot stars whose absolute flux is known from Hubble Space Telescope (HST) and IUE observations (Bertaux et al., 2006). A good knowledge of the absolute sensitivity is necessary for a correct quantitative analysis of emission measurements, while it is not necessary for stellar/solar occultations: a ratio of spectra allows to determine an absolute quantity of absorbers. 


\subsection{Geometry of nightglow/dayglow limb observations: the tangential limb mode}

Airglow spectroscopy and radiometry is a powerful method for investigating the physics of upper atmospheres of terrestrial planets (Venus, Earth, Mars). After the pioneering work of UV spectrometers on board Mariner 6,7,9 (Barth et al., 1971, 1992, and references therein), this technique was somewhat neglected for the following 30 years around Mars, except for rare observations in the extreme ultraviolet (EUV) with rockets and Earth orbiting observatories.

While dayglow was clearly measured and identified in the Mariner 6, 7, 9 observations, the first detection of nightglow in the atmosphere of Mars was made with SPICAM instrument on board the ESA MEX orbiter mission (Bertaux et al., 2005a,b). These successful observations are the result of the high sensitivity of SPICAM and specially dedicated spacecraft operations to optimize the geometry of limb observations, yielding more signal than the VEX and MEX nominal nadir-looking geometry around pericenter. This geometry is described in the following, with a sketch in Bertaux et al. (2006) JGR.

The axis of the SPICAV UV FOV is boresighted with the other optical instruments (VMC, VIRTIS, PFS), and aligned with the $+Z$-axis of the spacecraft, usually maintained pointed to the nadir for imaging near pericenter. Another standard attitude is with the TM antenna $(+X$ or $-X)$ directed to the Earth. In a third mode, the spacecraft is maintained in a fixed inertial attitude, a mode heavily used by SPICAM for star occultation measurements, and also for limb observations. In fact, on the dayside, there are two reasons to observe the aeronomical emissions at the limb: maximizing the integrated emission (Chapman factor), and eliminating the strong UV background of solar light scattered by the clouds of Venus which extends to high altitudes $(40-70 \mathrm{~km})$.

Planning a limb observation requires finding an inertial direction such that, during the orbital motion of VEX, the line of sight (LOS) will scan across the limb. In order to maximize the duration of the observation, we have designed a special type of observation in which the LOS does not cross the limb vertically, but rather skims the limb tangentially, with a minimum altitude, which may be selected (so-called tangential limb mode). Geometry tells us that, for any point $\mathrm{M}$ of the eccentric orbit, there are two LOS directions which will skim the limb tangentially, with a specified minimum altitude $Z_{\min }$, while the spacecraft orbits with a constant inertial attitude. They are contained in the two planes tangent to the sphere $R_{\mathrm{Venus}}+Z_{\mathrm{min}}$, which contain also the tangent to the orbit at $M$ (the velocity vector). One of them is selected to plan the observation once the point $\mathrm{M}$ is selected, according to the desired position of the tangent point at minimum altitude. One characteristic of this kind of observation is that several geometrical parameters of the LOS tangent point vary significantly and simultaneously during the observation (altitude, latitude, Solar Zenith angle [SZA]).

Given the success of this mode of observation on MEX (two discoveries, NO bands at night and aurora), we will use it as much as possible on VEX.

\subsection{The NO bands emission on the nightside}

One conspicuous Venus nightglow emission is the $\gamma$ and $\delta$ bands of nitric oxide (NO, 190-270 nm, respectively transitions $A^{2} \Sigma^{+}-X^{2} \Pi$ and $\left.C^{2} \Pi-X^{2} \Pi\right)$ produced when $\mathrm{N}$ and $\mathrm{O}$ atoms combine to produce NO molecules. This UV emission was first observed with Mariner 5 (Barth et al., 1968) and later with Pioneer Venus (Stewart et al., 1979) in the night airglow of Venus, and identified (Feldman et al., 1979; Stewart and Barth, 1979) as NO radiative recombination. It was proposed that $\mathrm{N}$ and $\mathrm{O}$ atoms are produced by EUV photo-dissociation of $\mathrm{O}_{2}, \mathrm{CO}_{2}$ and $\mathrm{N}_{2}$ in the dayside upper atmosphere, and transported to the nightside where recombination occurs, a mechanism later supported by 3D modelling (Bougher et al., 1990) and discussed thoroughly in terms of atmospheric circulation. There are no seasons on Venus, and the upper atmosphere at cloud top level $(65 \mathrm{~km})$ is rotating faster than the solid body (super-rotation), with a period of 4-5 days. The subsolar point is a region of upwelling, and the circulation of the thermosphere is a combination of both the rotation, and a general motion from subsolar to antisolar point, axisymmetric. Indeed, a maximum emission of NO night glow is found typically at $\mathrm{LT}=0200$, displaced from the antisolar point by super-rotation, with an intensity of $1.9 \pm 0.6$ kilo-Rayleigh $(\mathrm{kR})$, peaking at an altitude of $111 \pm 7 \mathrm{~km}$ (Stewart et al., 1980). Note that the sketch of super rotation of Venus in Bougher et al. (1997) is incorrect, if seen from the north pole, as is usual.

SPICAM on MEX has detected for the first time the same type of NO emission on the nightside of Mars (Bertaux et al., 2005a, b). Indeed, it is the major component of UV nightglow on Mars, together with $\mathrm{H} \mathrm{L} \alpha$ emission $(121.6 \mathrm{~nm})$. On Fig. 3 is displayed the spectrum recorded in the Mars nightglow. We expect that on Venus, the intensity will be larger, offering a powerful diagnostic tool to compare with models of general circulation of the atmosphere-thermosphere system. Of particular interest will be: the vertical distribution of the emission, which requires limb observations, and the latitude-Local Time distribution, which can be determined by nadir viewing, or during VIRTIS mappings of the nightside of Venus. It would allow us to disentangle the relative importance of the two systems of circulation: the super-rotation, and the solarantisolar circulation patterns.

\subsection{Auroral emissions on Venus}

Auroral emissions in planetary atmospheres are not restricted to the situation when energetic particles are precipitating along magnetic field lines, as is the case on 


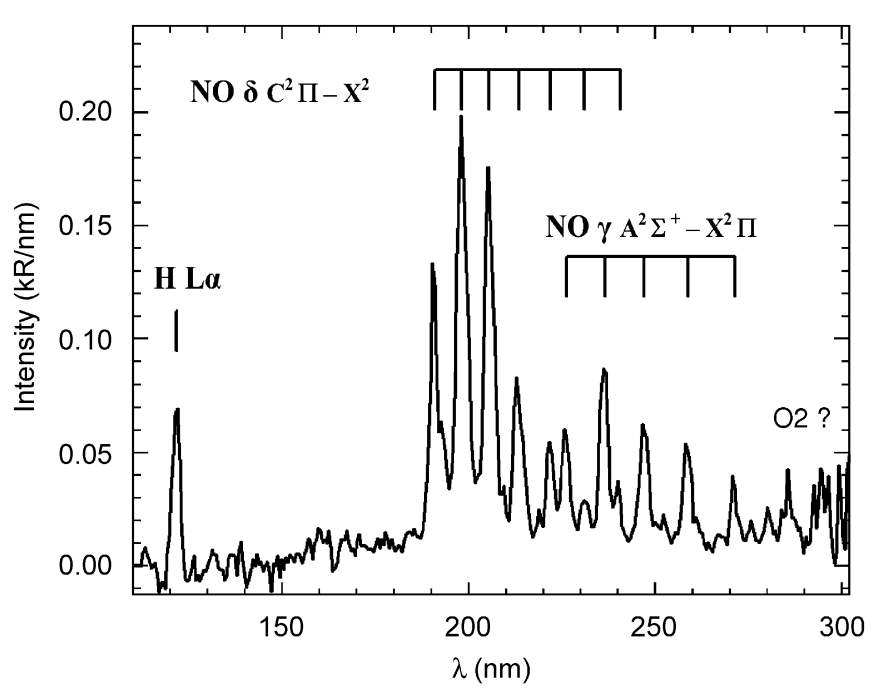

Fig. 3. Spectrum of the martian nightglow obtained with SPICAM/MEX at orbit 734 . Except for $\mathrm{L} \alpha$ at $121.6 \mathrm{~nm}$, all the observed lines coincide precisely with the main NO gamma and delta vibrational state transitions responsible for the Venus NO nightglow. The absolute radiometric calibration comes from SPICAM observations of hot UV stars. The total NO intensity is 2.33 kiloRayleigh $(\mathrm{kR})$, with a $74 \%$ contribution of the delta-band (and $26 \%$ from gamma-bands). The $(0,1)$ line of the delta band alone is, at $475 \mathrm{R}, 27 \%$ of the total delta bands. Spectral features above $280 \mathrm{~nm}$ could be attributed to the $\mathrm{O}_{2}$ Herzberg I system, also expected from the recombination of $\mathrm{O}+\mathrm{O}$ (taken from Science, Bertaux et al., 2005a, b).

Earth and all giant planets. Auroral activity has been found also on Venus, which has no magnetic field. On the Venus nightside, atomic $\mathrm{O}$ emissions at 130.4 and $135.6 \mathrm{~nm}$ appear in bright patches of varying sizes and intensities (Philips et al., 1986), which are believed to be produced by electrons with energy $<300 \mathrm{eV}$ (Fox, 1986): this is a manifestation of the interaction of the atmosphere of Venus with the solar wind, which produces an erosion of the atmosphere, that needs to be quantified to understand its evolution.

The potential of SPICAV to monitor these precipitations is illustrated by the first observation of an aurora in the Martian atmosphere, discovered by the UV spectrometer SPICAM on board MEX (Bertaux et al., 2005a, b). It corresponds to a new type of aurora not seen before in the solar system: it is unlike auroras at Earth and the giant planets, which lie at the foot of the intrinsic magnetic field lines near the magnetic poles, and unlike aurora at Venus, which is diffuse, sometimes spreading over the entire disc. Instead the Martian aurora is a highly concentrated and localized emission controlled by the crustal magnetic field anomalies.

In the observation quoted here, the SPICAM LOS was permitted to drift slowly across the nightside limb to search for any weak emission, since no Martian nightglow had been reported before, in the tangential limb mode. Fig. 4a (top) is a color-coded image of the time series of highresolution intensity spectra. The most obvious spectral features are the $\mathrm{H} L \alpha$ emission at $121.6 \mathrm{~nm}$, and the wellstructured band in the region $190-270 \mathrm{~nm}$ of the NO molecule, emitted when $\mathrm{O}$ and $\mathrm{N}$ atoms recombine, after having been produced by solar EUV photo-dissociation of $\mathrm{CO}_{2}, \mathrm{O}_{2}$, and $\mathrm{N}_{2}$ on the dayside and transported to the nightside, displayed on Fig. 8.

In Fig. 4b the nightglow signal integrated over the wavelength range of the NO bands (181-298 nm) is displayed as a function of time for the 5 spatial bins. The signal is more intense for spatial bins 3-5 than for spatial bins 1 and 2 because the FOV is wider and the source is extended. All curves show the same behaviour, almost identical to the variation of the $\mathrm{NO}$ emission observed 6 days later at orbit 734 (see above), which is explained by the variation of the altitude and the latitude of the Mars nearest point (MNP) when the LOS scans across the NO emitting layer, confined in the altitude range $60-80 \mathrm{~km}$, and more intense at large southern latitudes (around time $750 \mathrm{~s})$. There is, however, also a strong peak in all spatial bins between times 533 and 540 (increase by a factor 3 to 4 ), which has no equivalent during orbit 734 . The location of the emission could be located by a kind of "triangulation", using the 5 simultaneous FOV of SPICAV UV. It coincides precisely with the region of strongest crustal magnetic field B reported by Mars Global Surveyor (Acuña et al., 2001), and is the sign of electrons moving along the magnetic field lines, possibly connected to the interplanetary magnetic field at this location and time. The detected horizontal extent of this aurora is $30 \mathrm{~km}$ minimum, but it could be more extended along the LOS. The altitude of the observed emission is $129 \pm 13 \mathrm{~km}$. Still, it could extend more vertically, since it was scanned horizontally. The emissions in the auroral Mars spectrum are the CO $a^{3} \pi-X^{1} \Sigma^{+}$Cameron band between 180 and $240 \mathrm{~nm}$ (694 \pm 50 Rayleigh), long observed on the Martian dayside (Barth et al., 1971), as well as emissions associated with atomic carbon resonances and with the $\mathrm{CO} A^{1} \pi-X^{1} \Sigma^{+}$ Fourth Positive Group between 135 and $170 \mathrm{~nm}$, emissions associated with the $\mathrm{CO}_{2}^{+} B^{2} \Sigma^{u}-X^{2} \pi_{\mathrm{g}}$ doublet at $289 \mathrm{~nm}$ (71 \pm 42 Rayleigh).

Aurora on the nightside of Venus is one manifestation of the interaction of the solar wind with the ionosphere and the neutral atmosphere. This interaction is a major player of the erosion of the atmosphere of Venus, participating in its evolution through escape, and isotopic differential escape. As such, it is important to measure the aurora, in order to quantify this escape mechanism. The use of models like TRANSCAR predicting the intensities to be seen, when adapted to Venus, would be important in this respect.

\subsection{Airglow observations at the limb}

\subsubsection{Study of the ionosphere/thermosphere from $U V$ spectra}

The natural UV airglow of the atmosphere offers a definite possibility to study by remote sensing the ionosphere/thermosphere system and its temporal behaviour as a function of solar-wind parameters. Fig. 5 shows the 
a

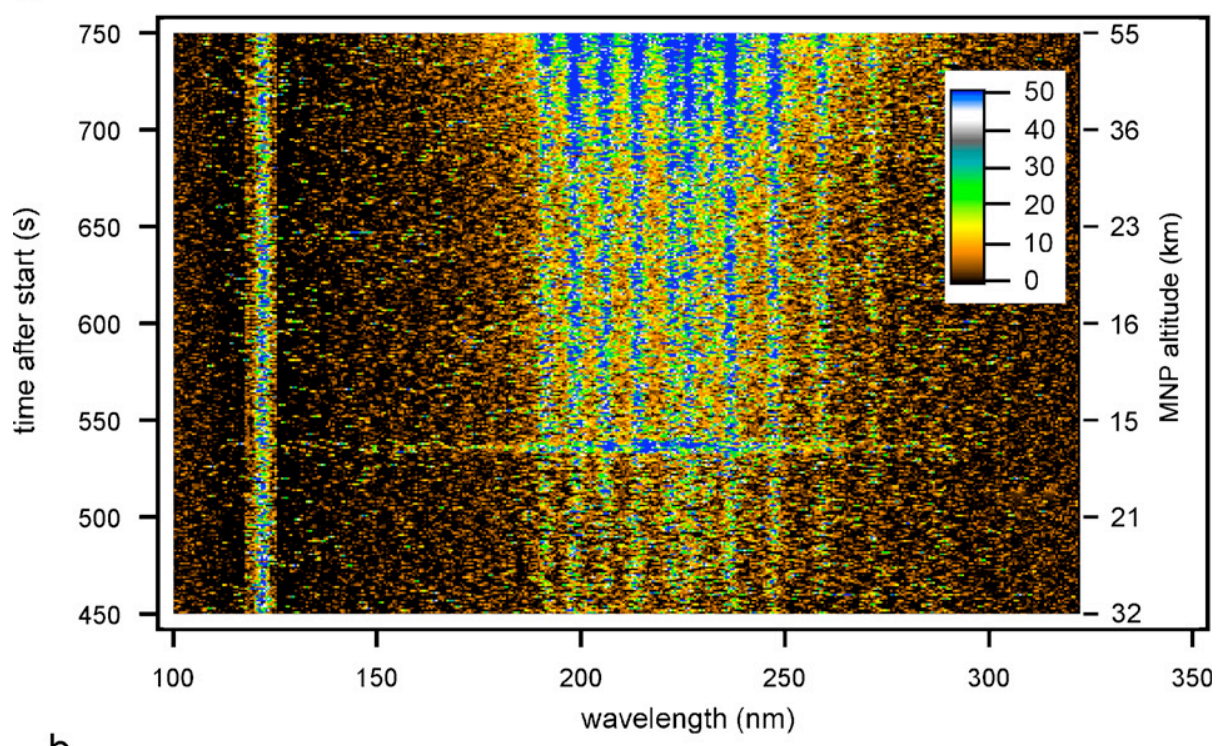

b

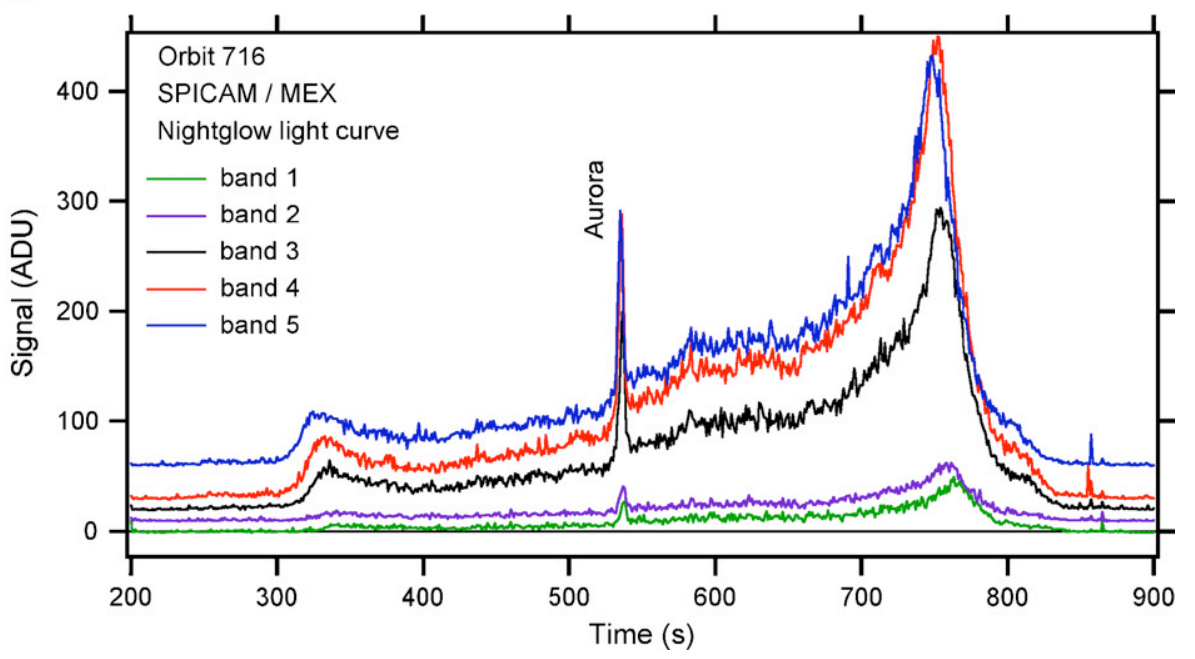

Fig. 4. (a) (top) Time variation of the Martian nightglow intensity spectrum recorded during the grazing limb observation with spatial bin 2 (narrow slit, spectral resolution $\sim 1.5 \mathrm{~nm}$, one spectrum per second, data subset from 450 to $750 \mathrm{~s}$ after the start of observation). It contains the H L $\alpha$ emission at $121.6 \mathrm{~nm}$ and a well-structured band (190-270 nm), identified as NO gamma and delta bands (Fig. 3). The intensity in ADU/pixel is colour-coded. Altitudes of the Mars Nearest Point of the line of sight are indicated at the right. At the time of the peak marked "aurora" on Fig. 4b, the spectra are obviously different from the typical NO spectrum. (b) Signal intensity for all 5 spatial bins as a function of time between 200 and 900 s, after the start of observations. Units are ADU per spectral pixel $=0.54 \mathrm{~nm}$, averaged from 181 to $298 \mathrm{~nm}$. There are 40 ADU per detected photon for the particular high voltage used here. The curves for spatial bins 2-5 have been vertically displaced for clarity (respectively by 10, 20, 30, 60 ADU). Spatial bins 3-5 have low resolution but high sensitivity, and bins 1 and 2 are less sensitive but have higher spectral resolution. A conspicuous spike marked Aurora is observed in all bins at time $535 \mathrm{~s}$. This is the time at which the spectra in Fig. 4a differ from the usual NO spectrum (figure taken from Nature, Bertaux et al., 2005a, b).

spectrum of dayglow recorded by SPICAM on the day side of the atmosphere of Mars (Leblanc et al., 2006). Some of these emissions have been observed on the dayside of Venus, as reported in Paxton and Anderson (1992). The main ionisable neutral constituent is $\mathrm{CO}_{2}$, and the $\mathrm{CO}_{2}^{+}$ transition $\left(B^{2} \Sigma^{u}-X^{2} \pi_{\mathrm{g}}\right)$ at $289 \mathrm{~nm}($ or $2890 \AA)$ is produced by photo-ionisation of $\mathrm{CO}_{2}$ from solar UV at $\lambda<69 \mathrm{~nm}$. The other band $\mathrm{CO}_{2}^{+}\left(A^{2} \Sigma^{u}-X^{2} \pi_{\mathrm{g}}\right)$ between 300 and $400 \mathrm{~nm}$ is produced by a combination of photo-ionisation and fluorescence scattering on $\mathrm{CO}_{2}^{+}$ions. The SPICAM UV long wavelength cut-off is at $320 \mathrm{~nm}$, which is enough to measure the (4.0) and 3.0) transitions of the $A-X$ band.
The intense Cameron band of $\mathrm{CO} a^{3} \pi-X^{1} \Sigma^{+}$observed at $190-270 \mathrm{~nm}$ is produced by a combination of photodissociation of $\mathrm{CO}_{2}$ by solar $\mathrm{UV}(\lambda<108 \mathrm{~nm})$, electron impact dissociation of $\mathrm{CO}_{2}$, and dissociative recombination of $\mathrm{CO}_{2}^{+}$. The variation of this band intensity with altitude was used to determine the vertical profile of $\mathrm{CO}_{2}$ above $120 \mathrm{~km}$, and from the scale height the temperature of the thermosphere was derived in the atmosphere of Mars (Stewart et al., 1972). This indirect method of determining $\mathrm{CO}_{2}$ could be validated with SPICAV UV channel, when a stellar occultation is performed on the dayside. If the pointing of VEX is as good as that of MEX, the small slit 


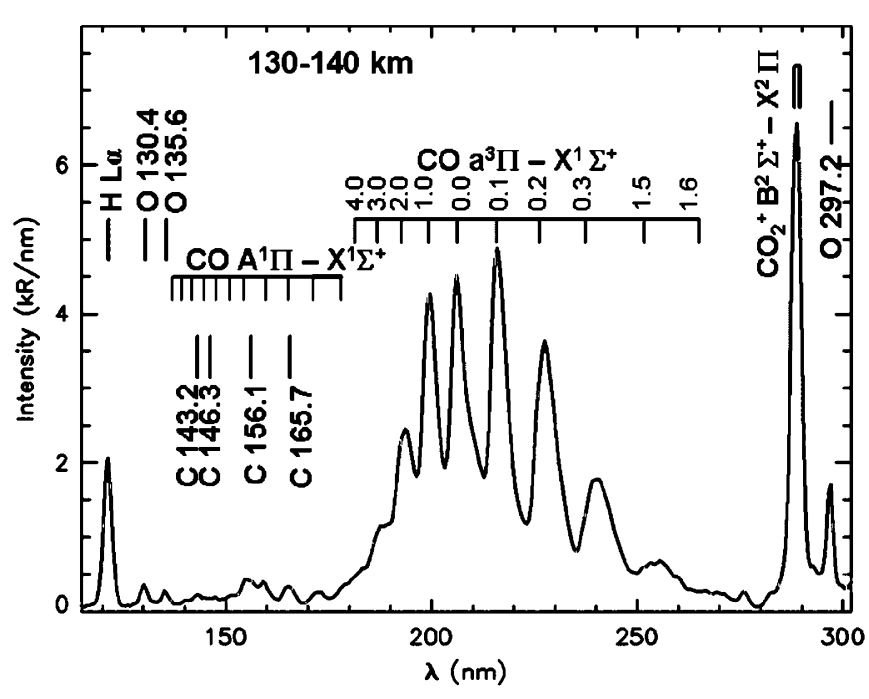

Fig. 5. Spectrum of dayglow recorded by SPICAM on the day side of the atmosphere of Mars (Leblanc et al., 2006).

of the FOV will be used, and the bright stellar spectrum would show up, superimposed on the general airglow only on a few lines of the CCD, and the analysis of the spectroimage would provide two independent sets of information on $\mathrm{CO}_{2}$ : direct measurement by absorptive occultation, and indirect $\mathrm{CO}$ Cameron band emission. Neutral $\mathrm{O}$ and neutral $\mathrm{H}$ vertical density profiles will be derived from the vertical variation of their resonance line at 130.4 and $121.6 \mathrm{~nm}(\mathrm{~L} \alpha)$, respectively, and the corresponding science is addressed in the next section.

Several atomic and molecular emissions have been reported in the far UV in the range 110-200 nm, either with the Hopkins Ultraviolet Telescope (Feldman et al., 2000), or with Galileo (Hord et al., 1991), looking directly at the sun illuminated disc. It should be understood that SPICAV might not be able to observe these emissions on the disc, but only at the limb. This is because the spectral range of SPICAM encompasses the near UV range $200-300 \mathrm{~nm}$, which is very bright (solar continuum on the clouds), and might damage the detector if a high gain is used. All these emissions will have to be observed at the limb, providing a vertical distribution.

\subsubsection{Hot oxygen corona and hot hydrogen corona of Venus}

The coronae of $\mathrm{H}$ and $\mathrm{O}$ atoms were reviewed by Paxton and Anderson (1992). While $\mathrm{CO}_{2}+h v \rightarrow \mathrm{CO}_{2}^{+}$is the main photo-ionization source, it is the $\mathrm{O}_{2}^{+}$ion, which is the most abundant below $200 \mathrm{~km}$, and $\mathrm{O}^{+}$above $200 \mathrm{~km}$. Recombinations of $\mathrm{O}_{2}^{+}$ions may produce hot $\mathrm{O}$ atoms, expanding in a hot atomic oxygen corona which was detected by Venera 11 (Bertaux et al., 1981) around Venus from the emission at $130.4 \mathrm{~nm}$ (Fig. 6), and the PVOUVS (Stewart et al., 1980). Models of this hot O corona (Nagy and Cravens, 1988 ) are in agreement with UV data. Note that it was not observed by Venera 12 flying by Venus only 4 days later; this would indicate a sporadic character (linked to solar wind variations?) of this hot corona. VEX will be able to

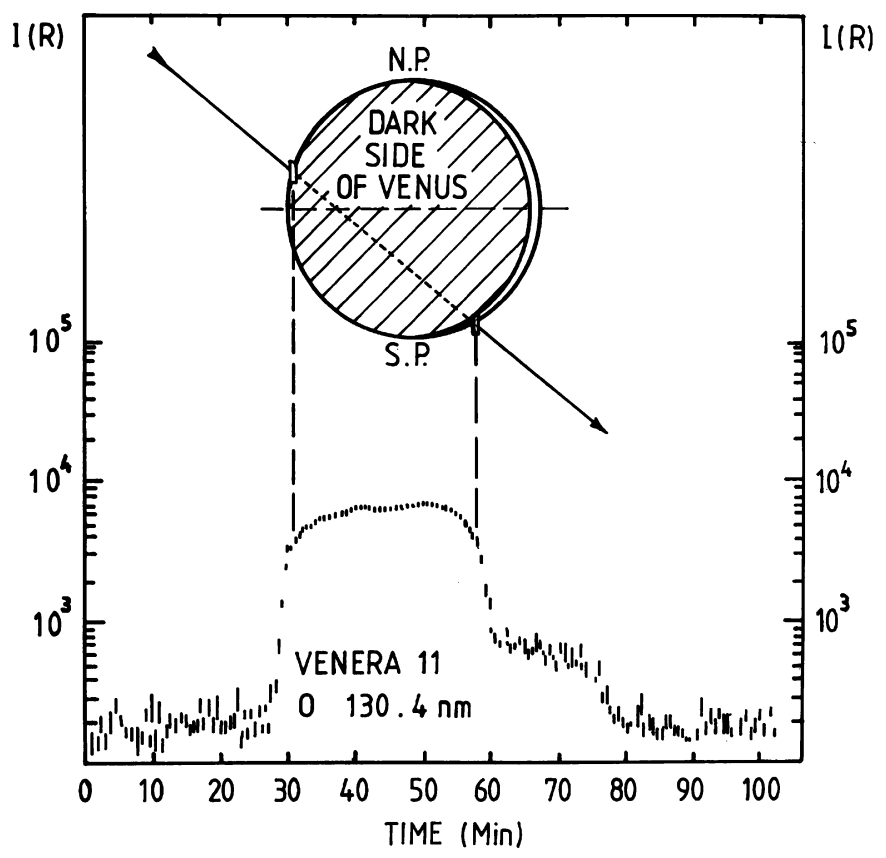

Fig. 6. The hot corona of $\mathrm{O}$ at $130.4 \mathrm{~nm}$, as seen from Venera 11 (Bertaux et al., 1981). The orbit passes behind the planet, the LOS is directed towards the reader. The bulge of the hot corona is located at high latitudes in the afternoon side. It was not seen by Venera 12 flying by Venus 4 days before in a similar geometry. Also, note the puzzling two-slopes behaviour of the intensity, not expected from models.

determine the time scale of the variation of emission from the hot $\mathrm{O}$ corona. Also, note in Fig. 6 the plateau in emission on the dayside limb, not expected from models. This hot corona may be an important source of $\mathrm{O}$ escape from the atmosphere of Venus, somewhat equilibrating the escape of $\mathrm{H}$ atoms responsible for the measured enrichment of $\mathrm{D} / \mathrm{H}$ ratio (a factor of $\sim 150$ ) detected by the Pioneer Venus mass spectrometer (Donahue et al., 1982) and confirmed in the IR in the lower atmosphere.

The $\mathrm{H}$ corona of Venus is peculiar, since it displays a radial distribution with two scale heights, one corresponding to the normal exospheric temperature $(\sim 300 \mathrm{~K})$, and the other at $\sim 600 \mathrm{~K}$ (Fig. 7), which dominates at larger distances, above $4000 \mathrm{~km}$ altitude (Bertaux et al., 1982). The common explanation is that the "hot" $\mathrm{H}$ population is produced by non-thermal reactions below the exobase (at $\sim 300 \mathrm{~km}$ altitude). However, a detailed analysis of the $\mathrm{H}$ vertical profile obtained from $\mathrm{L} \alpha$ measurements with Venera 11 and $12 \mathrm{UV}$ spectrometers (Bertaux et al., 1982) suggested that this population was absent below $\sim 4000 \mathrm{~km}$ (Fig. 8), casting some doubt about the region of origin and actual mechanism of production of this "hot" $\mathrm{H}$ population. The detailed vertical profiles obtained at the limb with high vertical resolution (which requires limb observations near the pericenter) will clarify this puzzle, and will help to quantify the present escape rate of $\mathrm{H}$ and $\mathrm{O}$ atoms by non-thermal mechanisms. The thermal escape, or Jeans escape, is easy to compute, once the exobase density and the exospheric temperatures are determined by the 


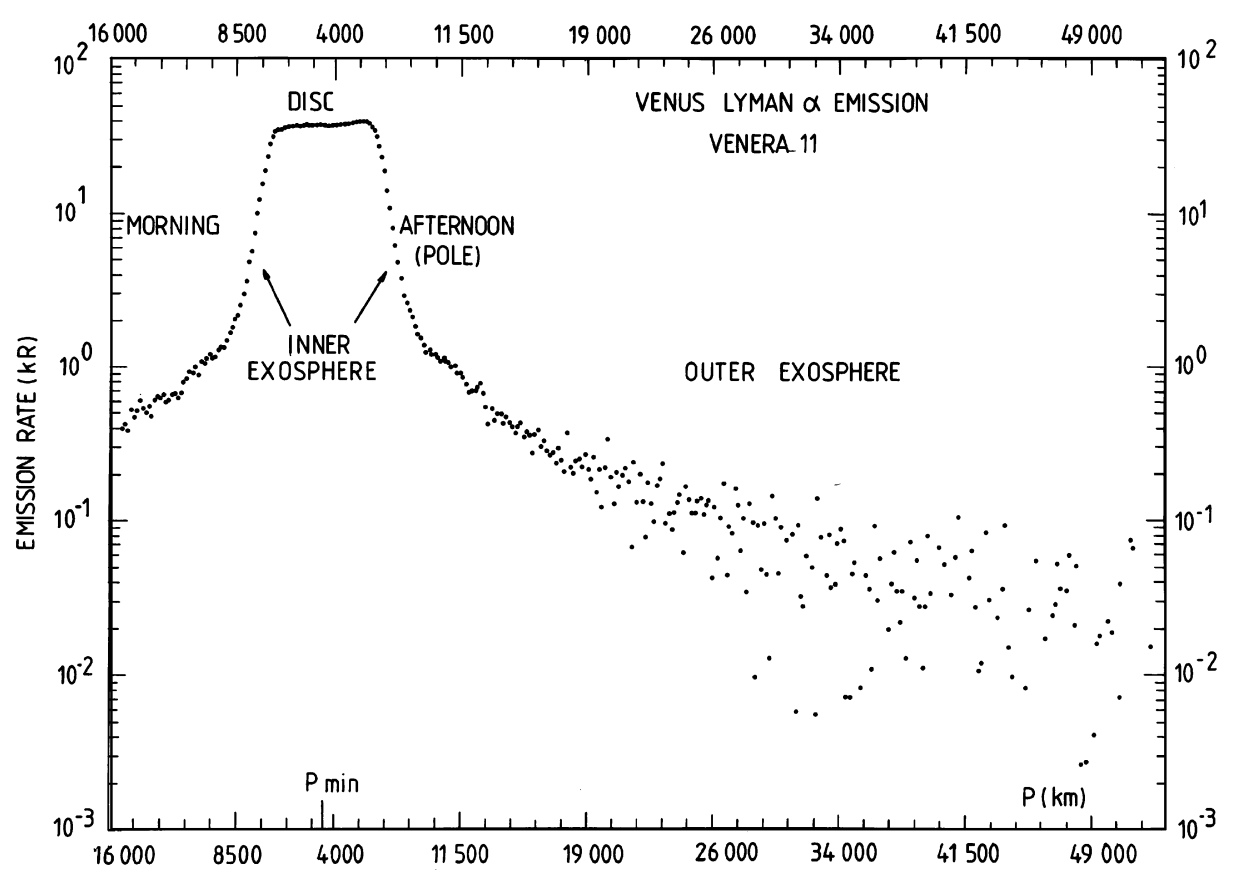

Fig. 7. The Hydrogen corona of $\mathrm{H}$ at $\mathrm{L} \alpha 121.6 \mathrm{~nm}$, from Venera111 and 12 measurements, as a function of impact parameter of the LOS. The two different slopes are seen on both sides of the planet: cold component below10,000 km (LOS distance to planet centre), hot component above $10,000 \mathrm{~km}$. Note the shoulder at $10,000 \mathrm{~km}$, not predicted by models (from Bertaux et al., 1982). Actually, it could be argued that the data suggests in fact three scale heights, with a change of slope for the cold component at about $8,000 \mathrm{~km}$ in the afternoon side.

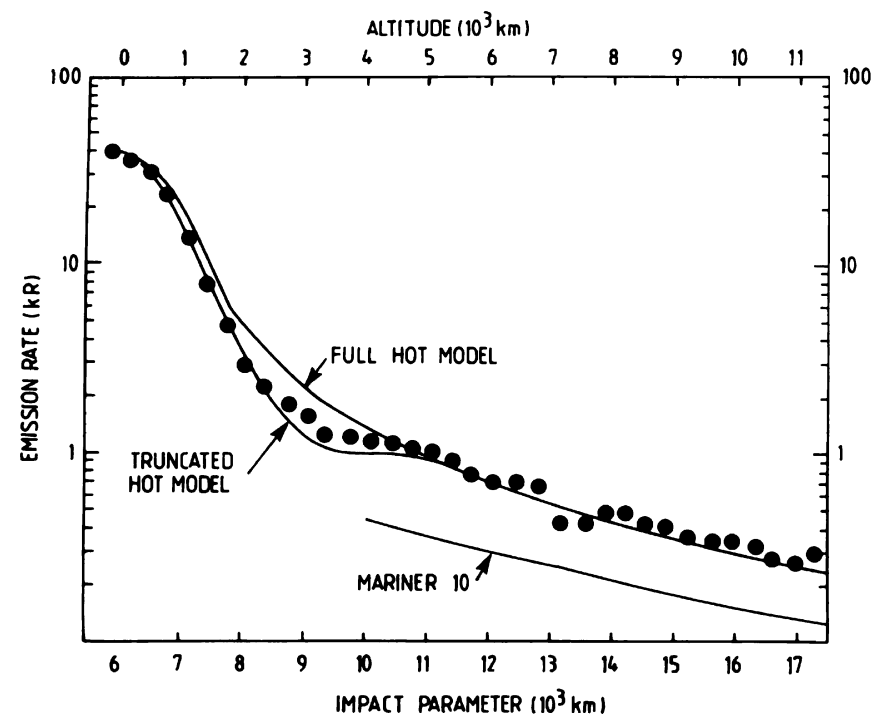

Fig. 8. Close up view of the hydrogen corona of at $\mathrm{H} \mathrm{L} \alpha 121.6 \mathrm{~nm}$. The hot component measured by Venera 11 in 1978 is twice more intense than measured by Mariner 10 in 1974, possibly due to higher solar activity. Note the deficit of the hot component around $4000 \mathrm{~km}$ altitude (from Bertaux et al., 1982).

SPICAV UV channel from the retrieved vertical altitude distribution of $\mathrm{H}$ and $\mathrm{O}$ atoms. An essential tool to interpret the $\mathrm{H}$ and $\mathrm{O}$ intensity data is a good radiative transfer model, such as the one was developed originally at Service d'Aéronomie by J.L. Bertaux and improved by E. Quémerais and J.Y. Chaufray.

\subsubsection{Atmospheric Escape and $D / H$ ratio}

At present there is only $3 \mathrm{~cm}$ of water in the atmosphere of Venus (if it were condensed to the liquid form), to be compared to $\sim 3 \mathrm{~km}$ on Earth. The current explanation is that water escaped from the upper atmosphere, because $\mathrm{H}$ atoms and $\mathrm{H}^{+}$ions can escape. The $\mathrm{D} / \mathrm{H}$ ratio is enriched by a factor of 150, compared to Earth (Donahue et al., 1982, 1999; De Bergh et al., 1991). This is explained by isotopic differential escape: $\mathrm{D}$ atoms are twice as heavy as $\mathrm{H}$ atoms, and are less prone to escape. Suppose that no D atoms could ever escape. The present content of D atoms would then reflect the total water that was in the atmosphere of Venus, that is $4.5 \mathrm{~m}$, very small compared to Earth. Therefore, it is absolutely crucial to measure the quantity of D atoms and HDO in the upper atmosphere of Venus, to estimate the rate of escape of D atoms. HDO will be addressed by the SOIR spectrometer (see below). For D atoms, the situation is not clear, with contradictory evidence. On one hand, the ion mass spectrometer on board Pioneer Venus orbiter detected a mass 2 ion in the upper atmosphere. First, it was interpreted as $\mathrm{H}_{2}^{+}$. Later, it was interpreted as $\mathrm{D}^{+}$ions (McElroy et al., 1982) with an abundance ratio $\mathrm{H}^{+} / \mathrm{D}^{+} \sim 100$, corresponding approximately to the lower atmosphere abundance ratio. On the other hand, no $\mathrm{L} \alpha$ emission from neutral $\mathrm{D}$ atoms has been detected so far. Both $\mathrm{D}$ atoms and $\mathrm{H}$ atoms produce a $\mathrm{L} \alpha$ resonant emission in the upper atmosphere, excited by the $\mathrm{H}$ solar $\mathrm{L} \alpha$ line. Since the wavelength separation $(H$, $121.566 ; \mathrm{D}, 121.533 \mathrm{~nm}$ ) is larger than the thermal width of each line, the radiative transfer of both types of $L \alpha$ are 
totally decoupled. If the $\mathrm{D} / \mathrm{H}$ ratio in the upper atmosphere ( $>120 \mathrm{~km}$ where $\mathrm{D}$ and $\mathrm{H}$ atoms are illuminated by the Sun without $\mathrm{CO}_{2}$ absorption) was similar to the lower atmosphere ratio of $\mathrm{HDO} / \mathrm{H}_{2} \mathrm{O}$, then from outside we could see the emission of $\mathrm{L} \alpha$ of $\mathrm{D}$ atoms, well separated from the $\mathrm{H}$ emission, at a level of more than $1 \mathrm{kR}$. However, Bertaux and Clarke (1989) used the IUE at high spectral resolution and found no D emission at all, with a much lower limit than $1 \mathrm{kR}$. Therefore, possibly there is a mechanism, which prevents $\mathrm{D}$ atoms from reaching the upper atmosphere. With $\mathrm{L} \alpha$ measurements of HST, Krasnopolsky et al. (1998) reported that the $\mathrm{D} / \mathrm{H}$ ratio in the upper atmosphere of Mars was lower by a factor of 10 than the $\mathrm{D} / \mathrm{H}$ ratio in the lower atmosphere. One possible explanation (Cheng et al., 1999) is that HDO is less photodissociated than $\mathrm{H}_{2} \mathrm{O}$, because of a smaller cross-section. This effect is probably insufficient to explain the discrepancy, and another explanation is shown to be also important (Bertaux and Montmessin, 2001): fractionation through condensation. HDO is more prone to condense in ice crystals than $\mathrm{H}_{2} \mathrm{O}$ from vapour phase, decreasing the $\mathrm{D} /$ $\mathrm{H}$ ratio when altitude is increasing in regions where the photo-dissociation rate is important. This effect is known to play an important role in the Earth's upper troposphere-stratosphere, where HDO is severely depleted just above the tropopause: it is likely present in Mars too, and SPICAV will determine the situation on Venus, mainly with SOIR solar occultations measurements of HDO and $\mathrm{H}_{2} \mathrm{O}$ simultaneously in the upper atmosphere.

Though the SPICAV UV channel has too little spectral resolution to distinguish $\mathrm{H}$ and $\mathrm{D} \mathrm{L} \alpha$ emissions, they could be tentatively separated from the vertical distribution of the combined intensities. While the $\mathrm{H}$ emission should present a smooth variation around the $\mathrm{CO}_{2}$ absorption limb (around $120 \mathrm{~km}$ ), because it is optically thick, the D $\mathrm{L} \alpha$ emission is optically thin, and the intensity doubles just above the limb. Any spike at the $\mathrm{CO}_{2} \operatorname{limb}$ in the vertical distribution of $L \alpha$ total intensity limb would be due to $\mathrm{D}$ atoms, and could reach $\approx 1 \mathrm{kR}$ according to modelling exercises. Therefore, both $\mathrm{D}$ abundance and $\mathrm{H}$ abundance could be determined in the upper atmosphere, and compared to $\mathrm{HDO} / \mathrm{H}_{2} \mathrm{O}$ measurements in the lower atmosphere in the IR, and also at high altitude with SOIR.

\subsection{Atmospheric vertical profiling by stellar occultation in $U V$}

\subsubsection{Overview of stellar occultations}

In the stellar occultation mode SPICAV UV will measure the vertical distribution of $\mathrm{CO}_{2}$, temperature, $\mathrm{SO}_{2}$, aerosols, and possibly $\mathrm{O}_{2}$ by using the technique of stellar occultation, which is very successful on MEX at the present time. The principle is quite simple (Fig. 9). Along its orbit, stars are occulted one after the other by the planetary limb opposite to the velocity vector. At a predetermined time, the spacecraft is oriented in such a way that the LOS of SPICAV UV is oriented towards a given star. Its spectrum recorded above the atmosphere (say, at $200 \mathrm{~km}$ ), unaltered by atmospheric absorption, serves as a reference spectrum. Then, while the spacecraft is maintained in a 3-axis, inertial attitude, the LOS to the star intersects deeper and deeper parts of the atmosphere, down

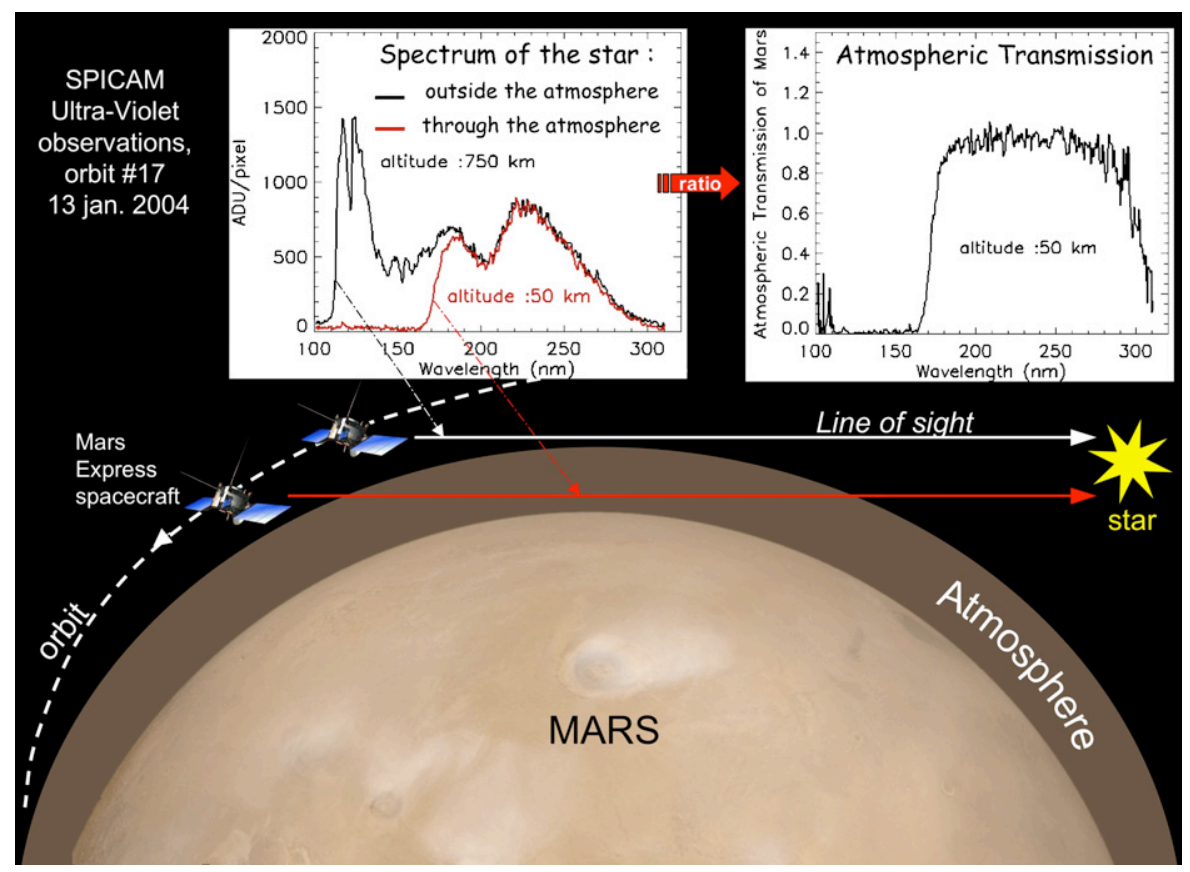

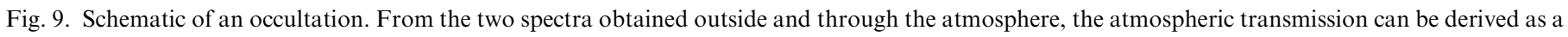

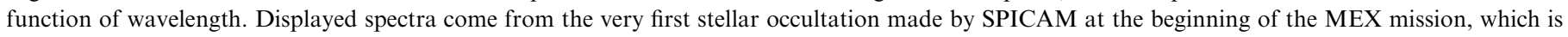

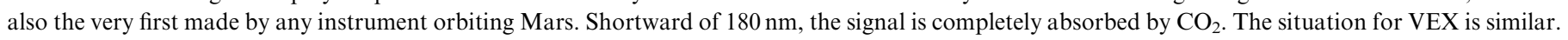


to total occultation. The stellar occultation technique offers three decisive features: an absolute concentration derived from a relative measurement (no need of instrument calibration, self calibrated method); excellent vertical resolution, for any distance to the planet (because the star is a point source); the accuracy of measurement altitude knowledge, at variance with limb emission methods, is independent of the attitude of the spacecraft. The LOS is entirely determined by the direction of the star in the sky (known) and the position of the $\mathrm{S} / \mathrm{C}$ in its orbit. Stellar occultations will be done preferably on the nightside of the orbit, to avoid contamination by the bright limb and will not affect the operation of dayside mapping instruments. Several (3-5) occultations per orbit are foreseen, the limiting factor being the spacecraft orientation as a resource to be shared between the various investigations of VEX.

Several features must be mentioned:

- The orbit of VEX is fixed in inertial space; when a star is occulted at one orbit, the same star will be occulted during the following orbits at the same latitude. Therefore, we have to find stars suitable to be occulted at various latitudes, and the occultations will occur in a repetitive manner, at variance with MEX, where the orbit and occultation patterns are changing quickly.

- Hot stars will be preferred, because they are brighter in UV. Their spectrum is flatter in UV than the solar spectrum.

- Both ingress and egress portions may be observed, since there is no need of a closed-loop tracking system.

- The UV spectrometer is used without the slit, and in spite of refraction that might change the apparent direction of the star, the stellar spectrum will still be recorded exactly.

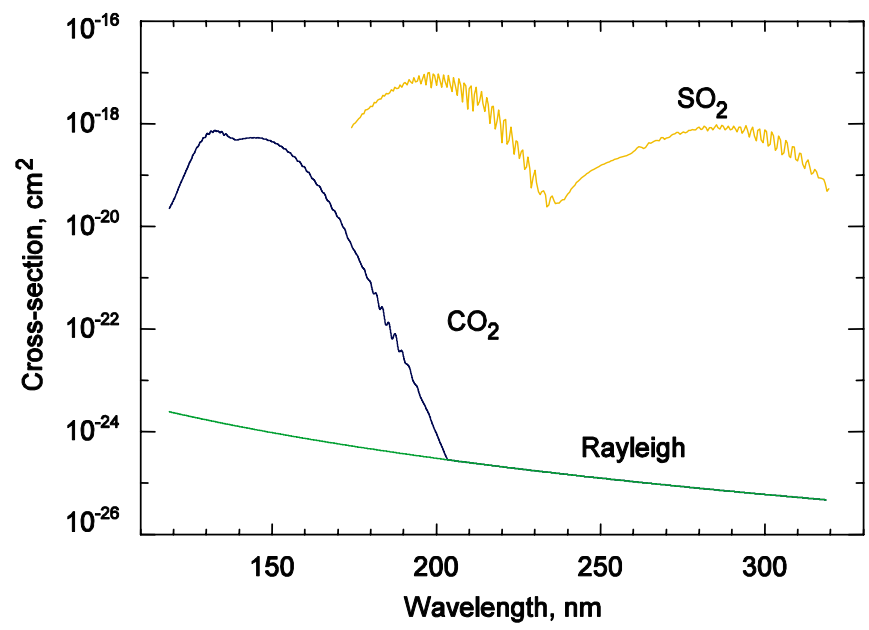

Fig. 10. Extinction cross-sections of $\mathrm{CO}_{2}$ and $\mathrm{SO}_{2}$, characteristic for the Venus atmosphere in the spectral range of SPICAV. The Rayleigh extinction of $\mathrm{CO}_{2}$ is also displayed. Aerosols/dust would add to the absorption everywhere in wavelength with a slope characteristic of the size of particles.
- Many opportunities of star occultations exist around pericenter, because Venus is much larger in the field of view, and occulting more sky. Therefore, we will request a certain number of dedicated occultations near pericenter.

Fig. 10 shows the absorption cross-sections of $\mathrm{CO}_{2}$ and $\mathrm{SO}_{2}$ in the $\mathrm{UV}$ as a function of wavelength. $\mathrm{O}_{2}$ is absorbing (Schumann-Runge bands) near the long wavelength edge of $\mathrm{CO}_{2}$, allowing the possibility to actually measure $\mathrm{O}_{2}$. Other absorbers are dust (Mie scattering generalized to non-spherical particles), and $\mathrm{SO}_{2}$. The atmospheric transmission simulations are presented in Fig. 11 for various tangential heights. Besides $\mathrm{CO}_{2}, \mathrm{SO}_{2}$ and dust profiles were assumed. Rayleigh extinction by $\mathrm{CO}_{2}$ was also included in the simulations. While on Mars, we can go down to $30-40 \mathrm{~km}$ most of time, on Venus the clouds will prevent probing below $60-70 \mathrm{~km}$. Because the $\mathrm{CO}_{2}$ cross-section presents an enormous dynamic range in the $\mathrm{UV}$, the $\mathrm{CO}_{2}$ absorption may be detected already at an altitude of $150 \mathrm{~km}$. For decreasing tangential heights, the $\mathrm{CO}_{2}$ manifests itself by a sharp cut-off that increases in wavelength, up to $\approx 200 \mathrm{~nm}$ at $z=80 \mathrm{~km}$ (Fig. 10). Longward of $200 \mathrm{~nm}$, the transmission spectrum is dominated by $\mathrm{SO}_{2}$, dust and $\mathrm{CO}_{2}$ Rayleigh extinction. The Rayleigh extinction above $200 \mathrm{~nm}$ can be computed from the $\mathrm{CO}_{2}$ line density determined below $200 \mathrm{~nm}$, and the remaining continuous absorption above $200 \mathrm{~nm}$ may be attributed to dust/aerosol for a determination of its vertical distribution and spectral characteristics in the UV, as illustrated by SPICAM/MEX measurements (Quémerais et al., 2006).

Results of stellar occultations from MEX have been presented at a number of conferences; see for reference the papers of Quémerais et al. (2006) on the actual data and inversion processes, Montmessin et al. (2006) for the cloud

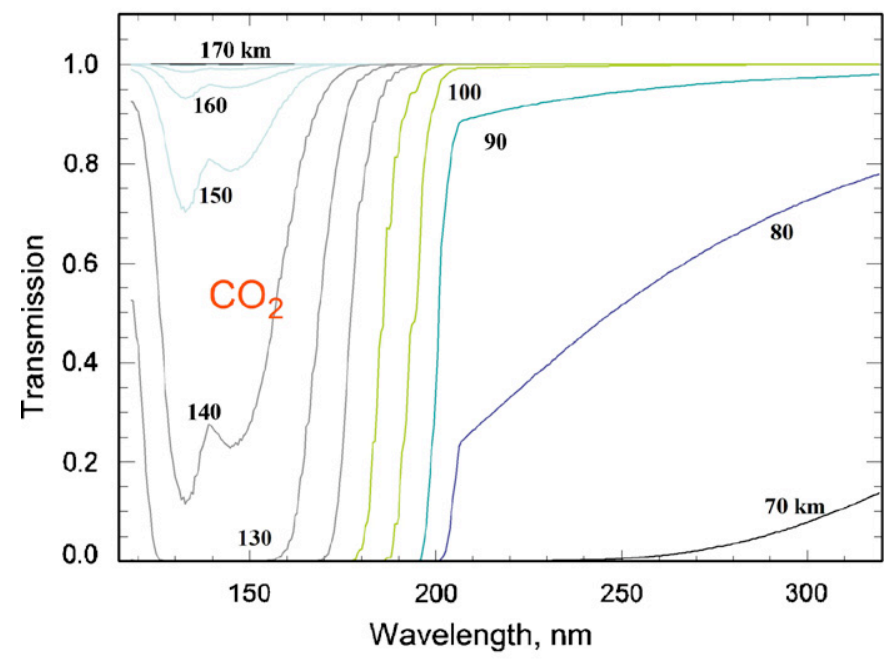

Fig. 11. Synthetic spectra of Venus atmosphere transmission in occultation for various grazing altitudes. $\mathrm{SO}_{2}$ is not taken into account in this simulation. 
and dust distributions, Forget et al. (2006) for the $\mathrm{CO}_{2}$ density and temperature measurements discussion, and Lebonnois et al. (2006) for the ozone results.

\subsection{2. $\mathrm{CO}_{2}$ density and temperature profile}

Once the line density of $\mathrm{CO}_{2}$ is determined from the transmission spectra for each altitude (one altitude per second), the local density $n(z)$ is determined from an Abel vertical inversion of the line density vertical distribution during one single occultation. Then, the hydrostatic equation allows temperature to be determined (Quémerais et al., 2006). Fig. 12 is a simulation of the retrieval of Venus atmospheric density in stellar occultation (Korablev and Bertaux, 2003). While PFS will be able to invert the IR spectra observed at nadir to determine a temperature profile in the lower atmosphere, SPICAV will provide the temperature and density profile at higher altitudes. On Mars with MEX, we have demonstrated the complementarity of both sets of measurements as a function of altitude (Fig. 13).

There is a known dependence of $\mathrm{CO}_{2}$ absorption cross section on the temperature $T$; the retrieval process will begin by a first iteration with an a priori profile $T(z)$ and corresponding choice of $\mathrm{CO}_{2}$ cross section. Then, the slant density $N$ and the local density $n$ are retrieved, and the scale height is derived, independently of a wrong choice of the cross section, providing a new guess for $T(z)$. A few iterations would allow convergence of the process. However, we should mention that this is not yet implemented for MEX in the SPICAM data reduction pipeline.

Though nighttime stellar occultations are preferred, it is important to keep in mind that some daytime occultations should also be performed using especially bright stars on the bright limb. On MEX we have proven that the pointing is so good that we can put the star image inside the $50 \mu$ slit of SPICAM. The day-night amplitude variation of the exospheric temperature (at top of thermosphere) is enormous on Venus (from $330 \mathrm{~K}$ down to $100 \mathrm{~K}$ at night) and is a crucial test for the validity of sophisticated thermospheric GCM models, such as the one developed by Bougher et al. (1990). SPICAV UV would offer a fair coverage of density/temperature profiles (local time, latitude, and solar activity: there are no seasons on Venus, and geography is assumed to be irrelevant for the upper atmosphere) with which the TGCM could be validated (or invalidated, and modified accordingly). Then, such a model could be used as a predictive tool for managing aerocapture/aerobraking operations in the future.

Finally, the unique ability of SPICAV UV to detect condensation clouds during the night should be mentioned. On the nightside of Mars, in more than $60 \%$ of occultations a distinct detached layer is found (Montmessin et al.,

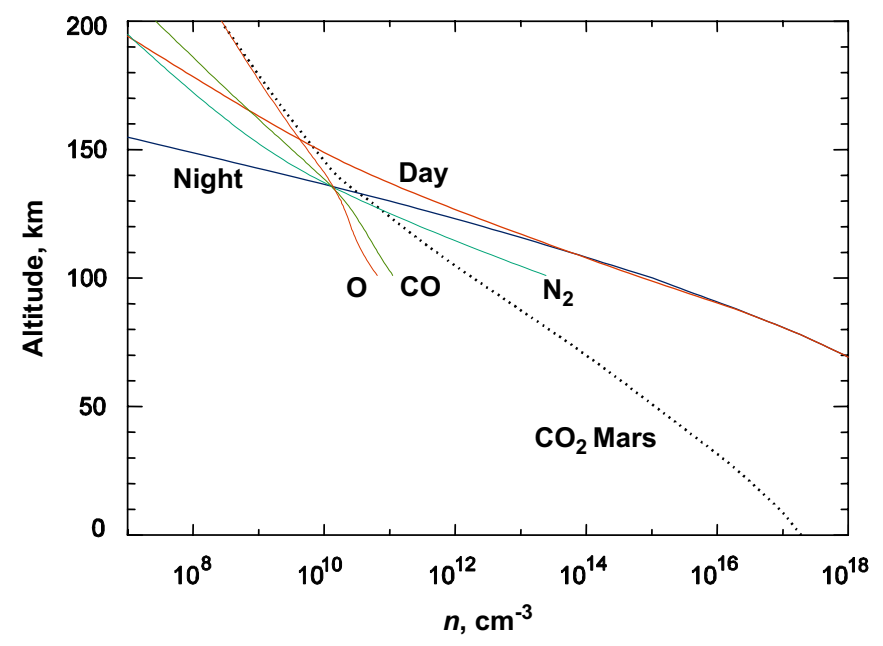

Fig. 13. A model of the Venus neutral atmosphere (solid lines) compared to Martian $\mathrm{CO} 2$ atmosphere, dotted line. Along with day and night $\mathrm{CO}_{2}$ profiles, day profiles of other most abundant constituents, $\mathrm{O}, \mathrm{CO}, \mathrm{N} 2$, are shown (after Kliore et al., 1985).
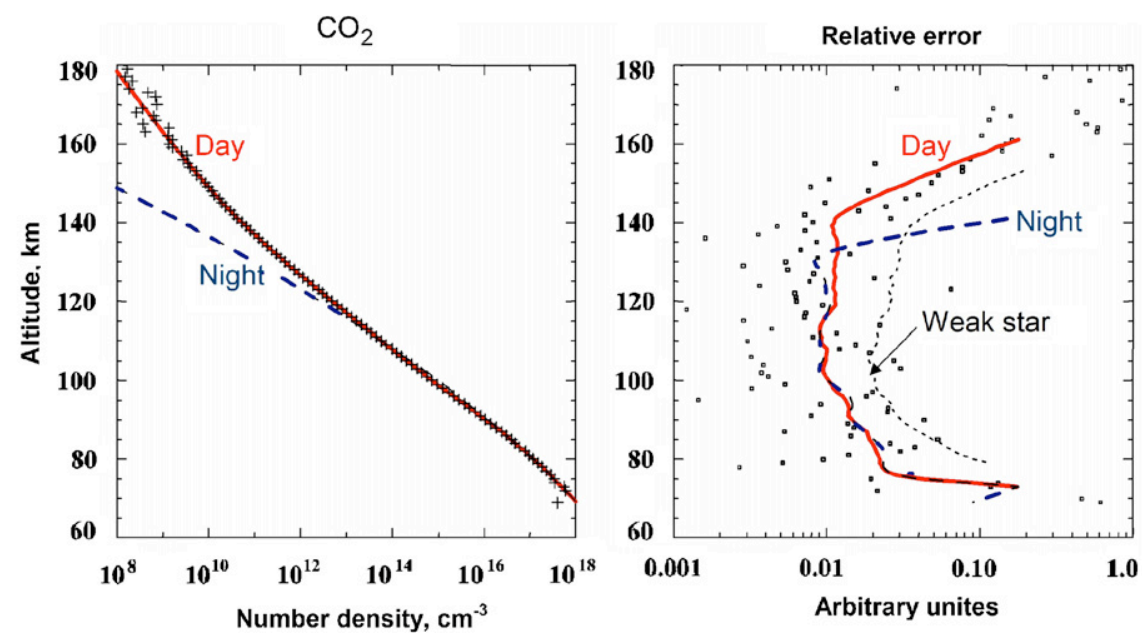

Fig. 12. Simulation of the retrieval of Venus atmospheric density in stellar occultation (Korablev and Bertaux, 2003). On the left are the model density profiles. Note the large difference of density between dayside (red curve) and nightside (dashed blue curve). The + are the retrieved densities after the spectral and vertical inversions. Relative errors are shown in the right panel. Scattered points illustrate an individual run; smoothed curves are averaged errors from 100 runs of the model for the dayside and nightside profiles. The modelling is performed for a bright UV star ( $\zeta$ Centauri). An error profile for the dayside and a weaker star (Vega) is also shown. The error is below $5-10 \%$ for most of the profile. 
2006): is it a dust or water ice cloud? In addition, SPICAM has seen several rare instances of a high altitude layer that is a $\mathrm{CO}_{2}$ condensation cloud, according to the temperature retrieved simultaneously from the $\mathrm{CO}_{2}$ vertical profile (Montmessin et al., 2006).

\subsection{3. $\mathrm{SO}_{2}$ vertical profiling}

$\mathrm{SO}_{2}$ is the most important minor constituent of the atmosphere of Venus. It combines with $\mathrm{H}_{2} \mathrm{O}$ to form the permanent thick cloud deck of $\mathrm{H}_{2} \mathrm{SO}_{4}$ droplets. The problem of its origin is still current. Thermochemical reactions with the surface minerals (Fegley et al., 1997) indicate that the $\mathrm{SO}_{2}$ equilibrium mixing ratio in the atmosphere at ground level should be of the order of 5 ppmv, with a time scale for equilibration of $20 \mathrm{Myr}$ at $735 \mathrm{~K}$. The fact that several descent probes found around $40-50 \mathrm{~km}$ a mixing ratio of $180 \mathrm{ppmv}$ has been used to suggest that $\mathrm{SO}_{2}$ is of volcanic origin, and may be fairly recent (w.r.t. the $20 \mathrm{Myr}$ time scale). However, the ISAV instruments on board the descent probes of Vega-1 and -2 have actually measured the vertical profile of $\mathrm{SO}_{2}$ in the clouds, below the clouds and almost down to the surface (Bertaux et al., 1996; Esposito et al., 1997). The $\mathrm{SO}_{2}$ mixing ratio was found to be $140-200 \mathrm{ppmv}$ at cloud level (different between Vega-1 and -2, but in overall agreement with previous in situ measurements), decreasing below, down to $\sim 10-20 \mathrm{ppmv}$ at $5-10 \mathrm{~km}$ of altitude (Fig. 14). Therefore, it indicates that indeed $\mathrm{SO}_{2}$ could be in equilibrium with the surface, with no requirement for a volcanic origin.
On the other hand, another observation has been interpreted as supporting a recent volcanic origin of $\mathrm{SO}_{2}$. It is the measured decrease of $\mathrm{SO}_{2}$ above the clouds over a few years, as reported by Esposito (1984) from Pioneer Venus UV spectra, interpreted as a sign of present decline of $\mathrm{SO}_{2}$, after an eruption occurring some years ago. However, this decline cannot be caused by recombining with surface rocks, in view of the time constants involved, and a volcanic origin requires a much more recent event than that required by surface disequilibrium. Is it possible to find a cause other than recent volcanism to explain the decrease of $\mathrm{SO}_{2}$ observed by Pioneer Venus? We will not attempt to do such an exercise here. We note, however, that the $\mathrm{SO}_{2}$ which is seen imprinted in the UV spectrum of cloud scattered sunlight is the uppermost part of the $\mathrm{SO}_{2}$, since it is rapidly photo-dissociated by solar UV above the clouds. It does not represent the bulk atmospheric $\mathrm{SO}_{2}$. Also, the fact that, in the clouds, the $\mathrm{SO}_{2}$ profiles are quite different for ISAV-Vega-1 and - 2 implies a large horizontal variability that may have a counterpart in time variability. At any rate, monitoring $\mathrm{SO}_{2}$ in various modes of observations (occultations, dayside nadir in UV, nightside viewing in the IR windows to probe lower altitudes) will be a prime objective of SPICAV and other instruments to obtain a better representation of its spatial and temporal variations.

\subsubsection{The case for Molecular Oxygen $\mathrm{O}_{2}$}

This molecule is the end result of $\mathrm{CO}_{2}$ photo-dissociation. Historically, $\mathrm{O}_{2}$ was discovered on Venus by its

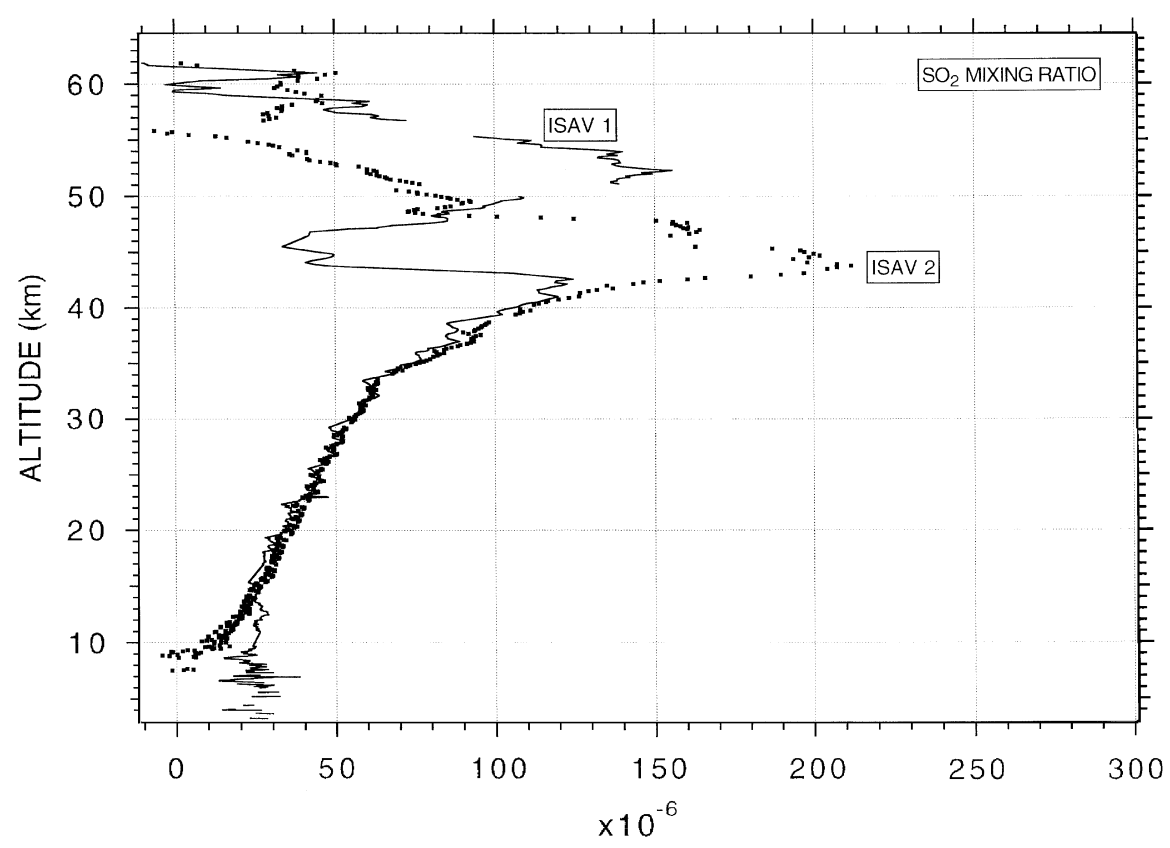

Fig. 14. ISAV measurements of $\mathrm{SO}_{2}$ vertical profile in Vega 1 and Vega 2 descent probes (Bertaux et al., 1996). The $\mathrm{SO}_{2}$ was measured by its absorption along a path of $1.7 \mathrm{~m}$, as recorded in the spectrum of the UV light of a Xenon flash. Above $40 \mathrm{~km}$, the two mixing ratio profiles are quite different at the two locations, linked to the vertical structure of the clouds, and the average value is in agreement with other previous measurements in this altitude range. Below $40 \mathrm{~km}$, both vertical profiles are very similar, exhibiting a steady decrease to lower altitudes, suggesting low values at ground levels, which would be compatible with chemical reactions with minerals. 
emission at $1.27 \mu \mathrm{m}$ by Connes et al. (1979) with Fourier transform high resolution spectra taken at Palomar. This emission occurs, day and night, when $\mathrm{O}$ atoms produced by EUV photo-dissociation of $\mathrm{CO}_{2}$ recombine to form the $\mathrm{O}_{2}$ molecule, much like the NO emission known on Venus and discovered recently by SPICAM on the nightside of Mars. This is quite a different process of emission than occurs in the Mars atmosphere, where this emission is produced by photo-dissociation of ozone. On the nightside of Venus, the recombination $\mathrm{O}+\mathrm{O}+\mathrm{M}$ produces also the Herzberg I and II system, discovered by Krasnopolsky and Tomashova (1980). These two emissions are produced right at the formation of the $\mathrm{O}_{2}$ molecule, which otherwise has totally escaped optical detection up to now, preventing any abundance measurement, and this is considered as a challenging question (Krasnopolsky, 2006). The best upper limit to $\mathrm{O}_{2}$ mixing ratio is $0.3 \mathrm{ppmv}$ near $60 \mathrm{~km}$ (Trauger and Lunine, 1983). Simulations performed for the Mars atmosphere suggest that the $\mathrm{O}_{2}$ molecule could be detected from its absorption in the Schumann-Runge bands $(170-210 \mathrm{~nm})$, provided that its mixing ratio is of the order of $\mathrm{O}_{2} / \mathrm{CO}_{2}=10^{-3}$. It seems therefore hopeless for Venus, unless the mixing ratio increases dramatically above $60 \mathrm{~km}$. The observation of the absorption band at $760 \mathrm{~nm}$ and/or at $1.27 \mu \mathrm{m}$ in the near IR by SPICAV IR, either toward the nadir, or in solar occultation, could be an alternate possibility.

\subsubsection{Profiling and characterising of atmospheric aerosols}

Occultation spectroscopy is probably the most sensitive remote technique of direct sounding the vertical structure of clouds and aerosols. Above the thick cloud there is some thin haze, the nature of which is not clear. We will use both stellar/solar occultations in order to characterize their quantity, their size distribution and possibly their nature. In solar occultation, the information about spectral continuum at distant spectral wavelengths will be a by-product of gaseous absorption retrievals. Once the slant atmospheric opacities at different wavelengths are obtained from occultation data, the aerosol extinction will be retrieved by Abel inversion. Then, using Mie theory (possibly adapted for non-spherical particles), a number of unknown parameters characterizing aerosol component can be extracted, e.g., size distribution $\left(r_{\text {eff }}, v_{\text {eff }}\right)$, and the real and imaginary parts of the refractive index of aerosol particles. Also, the vertical variation of key parameters, such as $r_{\text {eff }}$ and the particle number density will be retrieved. Observations of solar light scattered at the limb will be also useful in this respect, as it is for Mars at present with SPICAM. In both cases, SPICAM has determined at Mars (Montmessin et al., 2006; Rannou et al., 2006) a large variability of the Angström coefficient $\alpha$, which describes the continuous absorption of dust/aerosols as a power law of the wavelength, $\lambda^{-\alpha}$. This coefficient is related to the size of the particles, which are small when $\alpha$ is large $(\sim 3-4)$, submicronic (down to $0.1 \mu \mathrm{m}$ radius).

\subsection{Solar occultation measurements}

In addition to stellar occultations performed by SPICAV UV channel, all three SPICAV spectrometers will benefit from solar occultations by Venus. A special solar light entry port at $90^{\circ}$ from $+Z$-axis of the spacecraft (common axis of optical instruments, VMC, VIRTIS, PFS, SPICAV UV and NIR), can be pointed towards the Sun by orienting the spacecraft without disturbing the optical instruments. Pointing this auxiliary optical axis towards the Sun has proven on MEX to be no more difficult an attitude control task than keeping nadir orientation, or pointing to the stars. The angular diameter of the Sun as seen from Venus is $0.7^{\circ}$, but since the brightness of the solar disc is nonuniform in the UV, stable pointing is desirable (and well achieved on MEX). There is no precession of the orbit plane, and during one Venusian year, there are two periods of solar occultations. The latitude of the occultation varies between $+85^{\circ}$ and $-85^{\circ}$, as seen in Fig. 15, offering fair coverage of density/temperature profiles. Naturally, SOIR

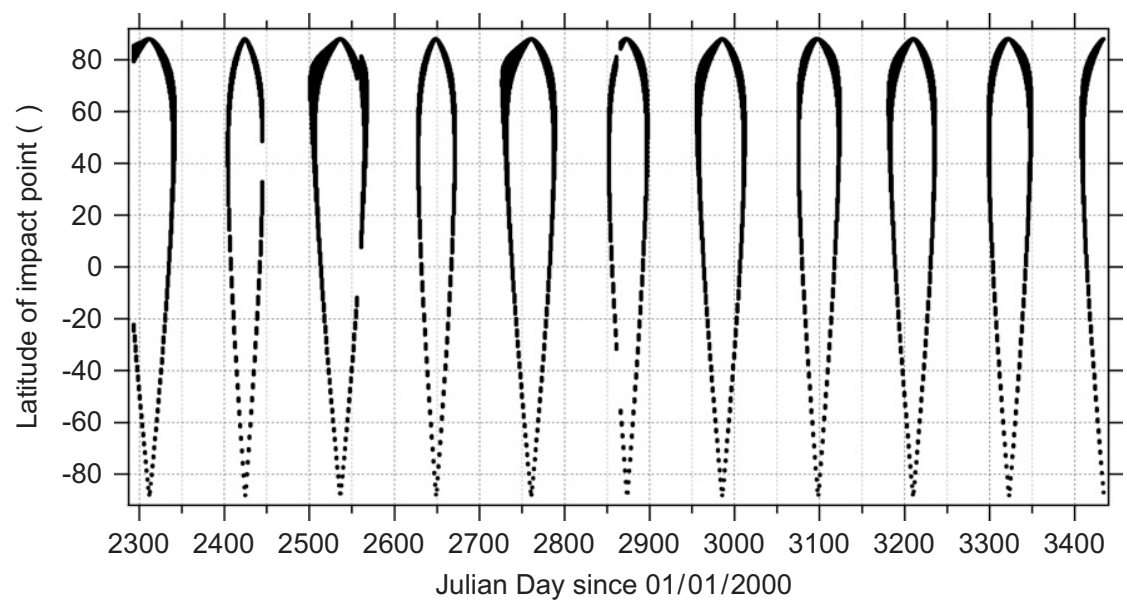

Fig. 15. Variation of the latitude of the Venus point where the solar occultation occurs over a 3-year period. There are two "seasons" of solar occultation per Venusian year. Time is counted in days since January 1st, 2000. 
will work essentially within these periods, and should have a highest priority.

Solar occultation spectroscopic sounding of a planetary atmosphere has several advantages compared to other methods of remote atmospheric sensing. The radiation of the Sun, which is an incomparably powerful source, traverses the atmosphere, providing the largest possible atmospheric path. The Chapman factor (airmass) reaches 40-45 for Venus. As for the case of stellar occultations, the measured spectra are compared to the unattenuated signal measured outside the atmosphere in the same sequence prior to occultation. No absolute calibration knowledge is necessary. A drawback of the solar occultation technique is that the atmosphere can only be observed when and where a sunset/sunrise occurs.

The vertical resolution of the UV channel in solar occultations is determined by the slit width in one direction and by a pixel height in the other, but also by the distance to the limb, which varies with time. Some periods are, therefore, more favourable for good vertical resolution. Assuming that the spectra will be integrated over two lines of the CCD, the FOV will be $1.5^{\prime} \times 1.5^{\prime}$, leading to a vertical resolution of 1.5 to $3 \mathrm{~km}$. If the spectrum from a single CCD line is considered, the vertical resolution could be twice as good for one coordinate (vertical or horizontal, depending on the orientation of the slit w.r.t. the limb). The FOV of the IR channel will be larger due to a simplified and miniaturized fibre optics that delivers solar light to the IR: about $4^{\prime}$, limiting the vertical resolution at the limb to $4 \mathrm{~km}$ for the best conditions.

The UV channel in solar occultation mode will perform the same measurements at the terminator as in the stellar occultation mode, with a better $\mathrm{S} / \mathrm{N}$ ratio, except below $190 \mathrm{~nm}$, where the solar spectrum is weak. Due to the high brightness of the Sun, deeper sounding will be possible in the upper cloud deck. The NIR channel, thanks to the solar occultation sounding, can measure vertical profiles of water vapour, as a complement to the more accurate measurements of SOIR.

As suggested by Don Hassler (private communication), slewing the spacecraft slowly when looking at the sun with SPICAV UV would produce a spectro-heliogram, thereby detecting active areas on the surface of the sun. This would allow monitoring solar activity on a face of the sun not visible from Earth during long periods; therefore, it would allow detecting well in advance active areas that will some days later become visible from Earth, providing an early warning for Space Weather activities.

\subsection{UV Nadir measurements}

\subsubsection{Dayside measurements}

On the dayside, the measured quantity is solar light scattered by the gaseous atmosphere (Rayleigh scattering) and by the particles in the clouds and hazes, modified by absorption of $\mathrm{CO}_{2}$ (at $\lambda<200 \mathrm{~nm}$ ), and by some other gaseous and particle absorption. When divided by the

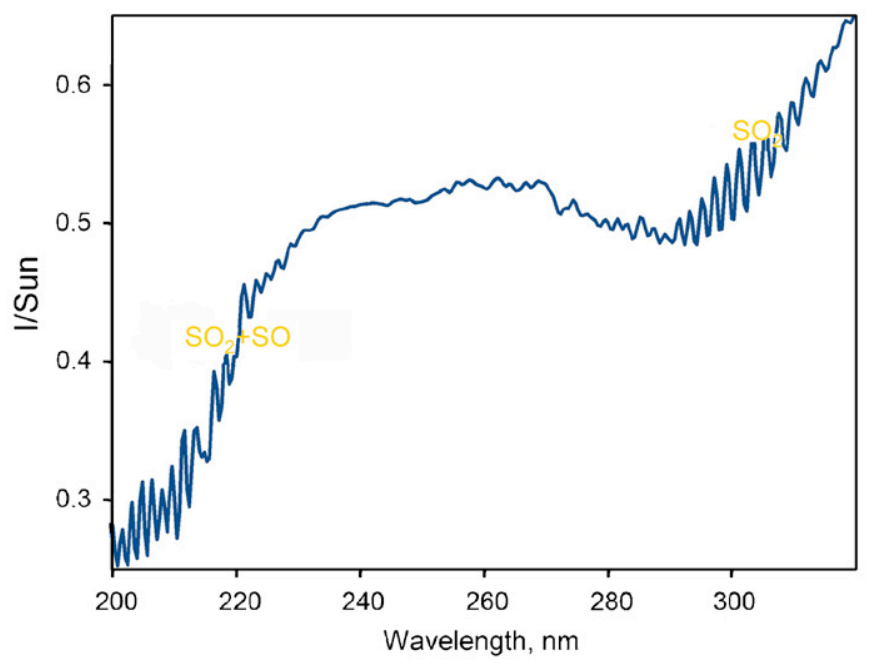

Fig. 16. Synthetic UV albedo spectrum of Venus at $0.4 \mathrm{~nm}$ resolution (computed by L. Zasova), showing the two regions of absorption due to $\mathrm{SO}_{2}$. SO is also absorbing in the region $200-230 \mathrm{~nm}$, with a complicated spectral structure.

incoming the solar spectrum, the nadir spectrum gives the albedo. In Fig. 16 is the result of a calculation by L. Zasova of the albedo of Venus in the UV, taking into account absorption by $\mathrm{SO}_{2}$ and $\mathrm{SO}$. As demonstrated with MEX, the UV sensitivity of SPICAV, blind in the UV $\lambda>320 \mathrm{~nm}$ and visible, will allow measuring accurately the absolute value of the spectral albedo between 110 and $310 \mathrm{~nm}$. SPICAV UV spectrometer will be regularly calibrated in flight observing the stars before occultations. The absolute solar spectrum outside the atmosphere is well known. Therefore, the albedo spectrum of Venus could be obtained with a good absolute accuracy and at a spectral resolution of $1.5 \mathrm{~nm}$. Then, comparing this spectral albedo to forward models using radiative transfer simulation codes (as is done for MEX, Perrier et al., 2006), a quantitative estimate of $\mathrm{SO}_{2}$, perhaps $\mathrm{SO}$, and other absorbers could be retrieved. The problem is not trivial, though, for a number of reasons. One is that the vertical distribution of particles may be poorly constrained (the occultation measurements will be useful, but not able to probe down to $\tau_{\text {vertical }}=1$ as would be desirable). The other is that the cloud deck is horizontally non-uniform. Indeed, the UV albedo of Venus is horizontally structured, a fact that allowed discovering the 4-day rotation period of the atmosphere of Venus at the cloud tops (Boyer and Camichel, 1961, 1965). In spite of many efforts, the exact nature of the UV absorber is still unknown (as discussed for instance in Bertaux et al., 1996). Is it the same substance, which also absorbs in the blue part of the spectrum? Is it in the gaseous phase, or in the cloud droplets? An answer to this irritating question could come from a detailed comparison of blue filter images from VMC and SPICAV UV measurements along track. While the retrieval from radiative transfer codes might be uncertain, horizontal variations will tell us much about the structure of the clouds. Finally, the nightside IR 
emission has been detected to be also highly structured horizontally: the IR radiation coming from the ground and lower atmosphere is strongly variable, suggesting a variable thickness of the cloud. A hole detected on the nightside could be observed 2 days later on the dayside, and it will be interesting to see if there is any correlation with the UV albedo.

\subsubsection{Nightside nadir measurements in the $U V$}

In the wavelength range of SPICAM UV (110-310 nm), the main emissions which have been detected are: $\mathrm{H} \mathrm{L} \alpha$, the $\gamma$ and $\delta$ bands of NO produced by radiative recombination, and auroral emissions, as discussed above. Here we discuss briefly the case of $H \mathrm{~L} \alpha$, which is directly connected to the thermosphere temperature. The thermosphere of Venus is somewhat extraordinary, because it varies from day to night in a wide range, from $\sim 300 \mathrm{~K}$ on the dayside to $100 \mathrm{~K}$ on the nightside. Therefore, on the nightside the whole upper atmosphere is "retracted" vertically. As a result, the coronal $\mathrm{H}$ is also retracted (and the density at $\sim 100 \mathrm{~km}$ is greatly increased), and there is little transport of solar $\mathrm{L} \alpha$ photons from the dayside (where they are scattered by $\mathrm{H}$ atoms) to the nightside. This factor, coupled with the very low temperature, which leads to a narrower $\mathrm{H}$ linewidth, makes the nightside of Venus very dark in $L \alpha$ as observed with Venera 9 and 10 (Bertaux et al., 1979). It is possibly the darkest place in the solar system in $\mathrm{L} \alpha$, much darker than the interplanetary medium at any rate. With VEX, we will have many opportunities to study in detail this weak emission on the nightside. Possibly the source of emission is mainly the backscattering of interplanetary photons. In such a case, it should vary as a function of Venus ecliptic longitude, reaching a maximum when the Doppler shift between Venus (including its orbital velocity) and the interplanetary flow of interstellar $\mathrm{H}$ is zero. In this respect, it would be useful to measure from time to time this $\mathrm{L} \alpha$ interplanetary emission from apocenter, in various directions.

Finally, we mention comets as interesting targets of opportunity, bearing in mind that Venus might be in a much better position in the solar system than the Earth to observe a comet. This was the case for comet Halley in 1986, a fact that changed the fate of the Soviet mission which was intended to place a big balloon payload (twice) in the atmosphere of Venus and two orbiters, but which was transformed into two fly-bys, each of them delivering one descending probe and a small balloon afloat for two days, the Vega-1 and -2 being targeted after the fly by to an encounter with comet Halley. Comet 45/P Honda Mrkos Pajdusakova will indeed come within 0.085 AU from Venus, and an observation campaign with VEX instruments will be organized.

\section{Near infra red spectrometer channel}

\subsection{The nightside IR emission of Venus}

A remarkable discovery of bright near-IR markings on the nightside of the planet (Allen and Crawford, 1984) has been made more then 20 years ago, but since then these features have not been observed by any spacecraft, except brief observations during the Galileo and Cassini fly-bys. At night it is possible to observe radiation in several spectral ranges (near 1, 1.23, 1.74 and $2.3 \mu \mathrm{m}$, Fig. 17) originating from different altitude levels below opaque clouds, down nearly to the surface (at $1.0 \mu \mathrm{m}$ ). Spectral

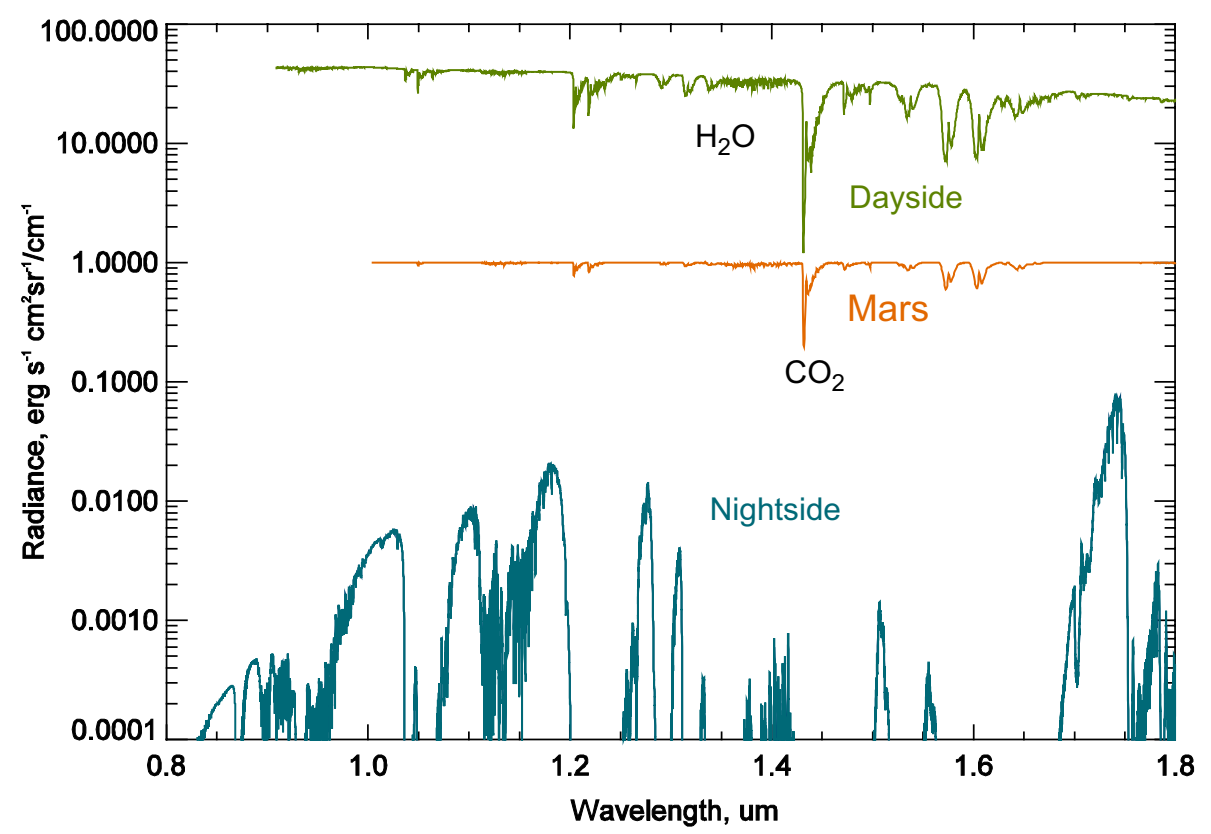

Fig. 17. Synthetic spectrum of the nightside emission from at spectral resolution of SPICAV IR compared to the spectrum of reflected solar radiation on Venus and on Mars. Note the dynamical range of 4.5 orders of magnitude between dayside and nightside observations. Cassini/VIMS spectrum (Baines et al., 2000) is shown for comparison. This nightside spectrum was computed by N. Ignatiev. 
analysis of this radiation gives the unique possibility to access remotely the deep levels of the Venus atmosphere. VIRTIS experiment will perform imaging and mapping in these windows, and SPICAV IR will analyse the nightside spectra at a high spectral resolution (comparable to the high spectral resolution channel of VIRTIS), with somewhat more flexibility and less data volume collection.

The SPICAV IR channel is derived from the very successful SPICAM IR /MEX, a miniature IR spectrometer based on AOTF technology. The scientific goals of SPICAV IR at Venus are as follows:

- Study of $\mathrm{H}_{2} \mathrm{O}$ and aerosol vertical distribution (solar occultations).

- Determination of the $\mathrm{H}_{2} \mathrm{O}$ abundance above the clouds (nadir, dayside).

- Monitoring of the 0.76 and $1.27 \mu \mathrm{m} \mathrm{O}_{2}$ emissions (limb).

- Observing the surface in $0.8-1.0 \mu \mathrm{m}$ "windows" (nadir, nightside).

- Measurements of $\mathrm{H}_{2} \mathrm{O}$ content near the surface (nadir, nightside).

\subsection{Technical description of the AOTF IR channel}

SPICAV IR is basically a single pixel spectrometer for the spectral range of $0.7-1.6 \mu \mathrm{m}$ with a spectral resolution of $3.5-8 \mathrm{~cm}^{-1}$, based on AOTF technology. It will sequentially measure the spectrum of reflected solar radiation from Venus on the dayside and the emitted Venus radiation in spectral "windows" on the nightside with a coarse spatial resolution of $10-15 \mathrm{~km}$ from VEX orbit. On MEX we have already demonstrated the operability of the AOTF in deep space missions (Korablev et al., 2001, 2006). For Venus the spectral range of the SPICAM AOTF MEX channel is extended down to $0.7 \mu \mathrm{m}$ with enhanced sensitivity in the entire range, and especially at $0.7-1.1 \mu \mathrm{m}$ to measure the radiance from the atmosphere below clouds and the surface of Venus on nightside. These measurements will complement low-resolution spectral mapping by VIRTIS in the same spectral range. One of the important goals of the SPICAV IR modification is to measure the $\mathrm{H}_{2} \mathrm{O}$ contents below clouds on the nightside. The dynamical range between dayside and nightside measurements is very large (see Fig. 17), and to accommodate it both the increase of the sensitivity of the instrument and much longer exposures are required. As in SPICAM/MEX, the chosen configuration of the AOTF conserves both polarizations allowing therefore spectropolarimetry measurements. A fiber-coupled solar entry port allows profiling the Martian atmosphere in solar occultations (Fig. 1). The AOTF devices, based on Bragg diffraction of an entrance beam on the ultrasonic acoustic wave excited within a crystal, are rather new. However, they offer the potential of reaching a resolving power larger than 1000 , ample to measure $\mathrm{H}_{2} \mathrm{O}$ towards the nadir by scanning the absorption line at $1.38 \mu \mathrm{m}$ in the solar reflected spectrum. There are no moving parts such as a chopper.

The main SPICAV IR AOTF spectrometer characteristics are summarized in Table 3. As in the SPICAM MEX instrument, the AOTF IR spectrometer of SPICAV is mounted alongside the UV spectrometer. The AOTF is made of crystalline tellurium dioxide, $\mathrm{TeO}_{2}$ used in the socalled non-collinear configuration. It produces two polarizations of the diffracted light. The diffracted beams are

Table 3

\begin{tabular}{|c|c|c|}
\hline & Short-Wavelength Range & Long-Wavelength Range \\
\hline Spectral resolution & $\begin{array}{l}0.42 \mathrm{~nm} \text { at } 630 \mathrm{~nm} \\
1.44 \mathrm{~nm} \text { at } 1050 \mathrm{~nm}\end{array}$ & $0.55 \mathrm{~nm}$ at $1.05 \mu \mathrm{m}$ \\
\hline FOV & $2^{\circ}$ circular & 1.5 \\
\hline Telescope & Lens type, Ø12 mm; focal length $40 \mathrm{~mm}$ & \\
\hline AOTF & $\mathrm{TeO}_{2}$ & \\
\hline $\mathrm{RF}$ frequency range & $140-250 \mathrm{MHz}$ & $80-140 \mathrm{MHz}$ \\
\hline \multirow[t]{4}{*}{ Detector } & Two bicolor diodes (Hamamatsu K3413-05) & \\
\hline & 1 stage TE cooled to $-15^{\circ} \mathrm{C}$ & \\
\hline & Silicon & InGaAs \\
\hline & $2 \times 2 \mathrm{~mm}$ & $\varnothing 0.5 \mathrm{~mm}$ \\
\hline Transmission of optics & $\sim 20 \%$ & \\
\hline Power consumption & $1.5 \mathrm{~W}$ average at $28 \mathrm{~V}$ & \\
\hline Data rate: & 6.2 kbyte per spectrum & \\
\hline Dimensions & $220 \times 85 \times 65 \mathrm{~mm}$ (not including DC/DC and DPU) & \\
\hline Mass of the IR unit & $700 \mathrm{~g}$ (not including DC/DC and DPU) & \\
\hline
\end{tabular}


deflected at a small angle w.r.t. the undiffracted, "white" output beams. If the acoustic wave is not applied to the crystal, there are only two undiffracted beams, and when the acoustic wave is turned on ( $\mathrm{RF}$ is turned on) two diffracted beams appear. These two weak useful beams should be, therefore, dependably separated one from another and from the strong undiffracted beams, which contain most of the spectrum. The active zone of the AOTF crystal is $25 \mathrm{~mm}$. Two detectors with proximity lenses record the ordinary and the extraordinary beams. The undiffracted central beams are captured by a light trap.

The new requirements concerning spectral range, sensitivity, and dynamic range finally led to substantial modifications of the AOTF spectrometer w.r.t. its MEX version (Korablev et al., 2002b,c). The details of the optical and electronics design, and the new AOTF will be described elsewhere; here we only summarize the new features of the instrument.

To extend the spectral range from $1-1.65 \mu \mathrm{m}$ to $0.7-1.65 \mu \mathrm{m}$, the AOTF was modified by adding a second, short-wavelength range actuator, to cover the part of the spectrum from 0.7 to $1-1.1 \mu \mathrm{m}$. A single $\mathrm{TeO}_{2}$ AOTF crystal, similar to that of the SPICAM MEX instrument, is equipped with two piezoelectric actuators, one for the range of $0.7-1.1 \mu \mathrm{m}$, and another for the range of $1-1.7 \mu \mathrm{m}$. The actuators are fed by two separate RF amplifiers, using a common programmable RF synthesizer with two outputs (f and 2f). For the purpose of testing, the spectral range of the short-wavelength channel attains the wavelength of red $\mathrm{HeNe}$ laser at $633 \mathrm{~nm}$, but the AOTF efficiency at this extreme wavelength is low. Furthermore, the sensitivity of InGaAs photo-detectors below $1.1 \mu \mathrm{m}$ is reduced, and another detector is necessary to cover the short wavelength channel. In place of Hamamatsu InGaAs G5832-11 $\varnothing$ $1 \mathrm{~mm}$ photodiodes used on MEX we used the so-called two-color sandwich detectors (Hamamatsu K3413-05). The electrically cooled detector consists of a silicon $2 \times 2 \mathrm{~mm}$ photodiode on which is superimposed a InGaAs $\varnothing$ $0.5 \mathrm{~mm}$ photodiode.

Several factors allowed improving the sensitivity and the dynamic range in the new design. First, the silicon photodiode and the smaller InGaAs photodiode are less noisy than the original 1-mm InGaAs detector. Further handling of the signal is completely new. Each photodiode feeds current to low noise integrating preamplifier IVC102 (TI and Burr-Brown). Preamplifiers are followed by variable amplification stages followed by an integrating amplifier. Spectral points are measured sequentially and the detailed sequence for a single measurement of one spectral point is found in Bertaux et al. (2006).

In the optical scheme, a simplification of the entry telescope was achieved by enlarging the field-of-view angle from $1^{\circ}$ to $2^{\circ}$. The use of long integration times required special attention to reducing stray light in the spectrometer, and the use of a smaller detector required more care in focusing of the output beams. The NIR channel uses for solar occultations a solar entry port near the solar entry port of the UV spectrometer; an optical fiber delivers the solar light to the NIR objective. Entry optics of this fiber provides an angular FOV of about 4 arcmin. A collimator lens at the output of the fiber and a $45^{\circ}$ flat mirror mounted at the baffle of the NIR objective complete the design of the solar entry for the IR (Fig. 1).

The frequency of ultrasonic excitation of the AOTF is controlled by software, so we are free to choose the AOTF wavelength within the spectral range. Then only the most important and interesting parts of the spectrum may be measured in nominal mode with desired sampling. During the first orbits, we will use the full range of the AOTF, to acquire full spectra at maximum sampling, at the expense of longer sampling intervals. Then, after a first analysis, we will restrict the spectra to the most interesting parts, with a great variety of spectral samplings, accomplished with three windows and a number of dot spectral points to cover the full range. Both the ordinary and extraordinary beams at the output of an AOTF crystal can be analysed simultaneously with two identical detectors, and the polarization of the incident light can be measured with high accuracy (Glenar et al., 1994). Polarimetry measurements enable a characterisation of the properties of atmospheric aerosol component (Santer et al., 1985). The spectral measurements by SPICAV NIR channel could be used for cross-validation of VIRTIS and PFS data.

\subsection{Operations and capabilities}

In order to illustrate the capabilities on VEX, we show some results obtained on MEX with the AOTF spectrometer $(1-1.7 \mu \mathrm{m})$. Due to the nature of the AOTF sequential measurements from a spacecraft orbiting the planet, each measured spectral point generally corresponds to a different spot at the surface. The FOV of $1^{\circ}$ corresponds to $\sim 5 \mathrm{~km}$ from the MEX pericenter, and it is, therefore, desirable to limit the overall duration of the measurement of the whole spectrum to 5-10 s. However, the full scan of the spectral range at fine spectral sampling (3-4 points per element of the AOTF spectral resolution) requires almost 4000 points, and with a reasonable integration time $(6 \mathrm{~ms})$ leads to $24-\mathrm{s}$ measurements. But the AOTF can be randomly tuned to any wavelength within the spectral range allowing us to minimize the measurement time without compromising the science return. The IR spectrometer can be programmed to scan up to 3 windows, and to determine for each window the bounds and the spectral sampling. Furthermore, several sets of predefined "dots" are available to characterize the spectral continuum (albedo of the surface, reflectance at limb, extinction, etc.) During the commissioning phase of MEX we first recorded a number of complete spectra, and then we defined the parameters of the optimized windowdot sets for different observation modes (see Fig. 18). Also, such parameters as gain, AOTF power, etc., were optimized. 

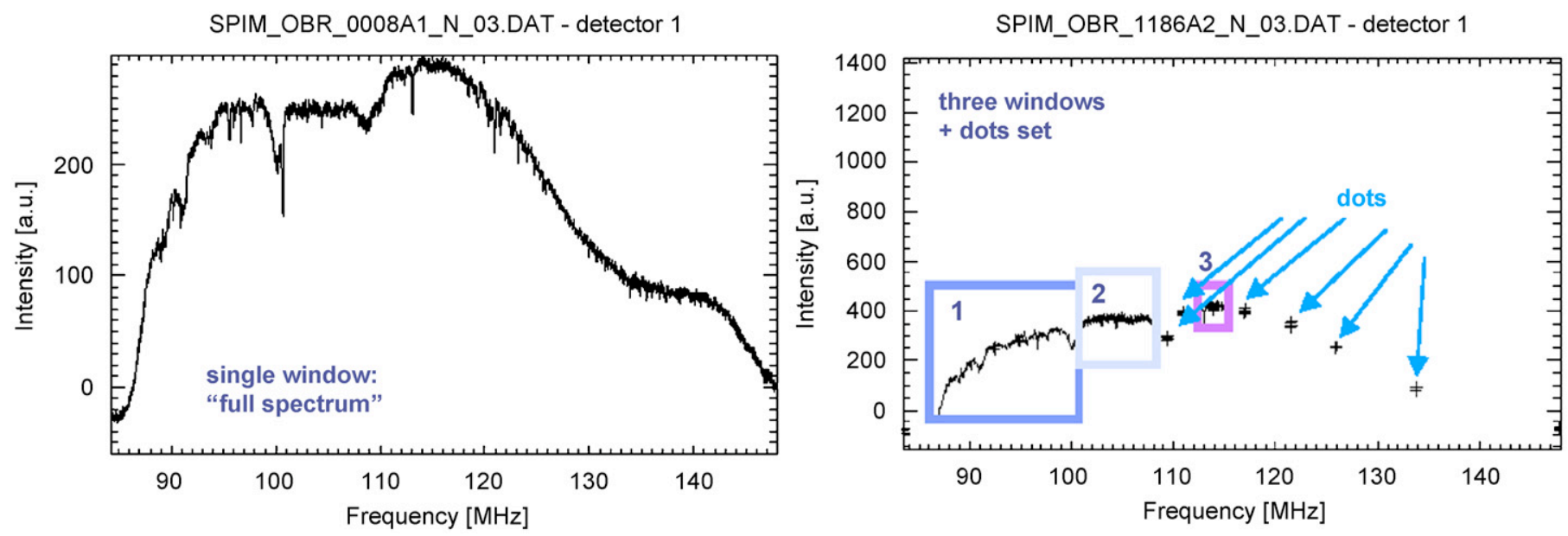

Fig. 18. An illustration of SPICAM IR channel operation modes. Left: a "full spectrum" when the AOTF driving frequency spanning the entire range and the AOTF is sequentially tuned to all wavelengths from 1 to $1.7 \mathrm{~mm}$. With a fine sampling such a sequence requires several seconds (12-24 s, depending on integration time etc.) to be completed. Right: Optimized sequence, in which only the interesting portions ("windows") of the spectrum are scanned at maximal sampling, less important are scanned with reduced sampling, and the rest of the spectrum is characterized by only few continuum points ("dots").

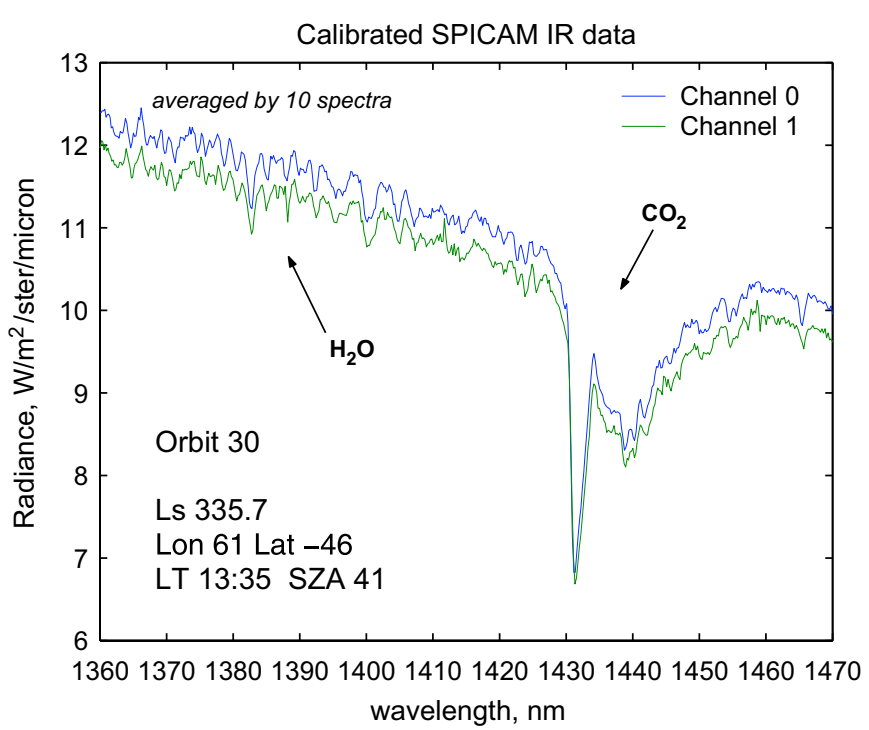

Fig. 19. A portion of a spectrum, showing the vicinity of $\mathrm{H}_{2} \mathrm{O}$ absorption band at $1.38 \mu \mathrm{m}$, and the adjacent $\mathrm{CO}_{2}$ band at $1.43 \mu \mathrm{m}$. For the purpose of clear representation of a complicated $\mathrm{H}_{2} \mathrm{O}$ band, 10 subsequent spectra (1 sec. each) of Orbit 30 of MEX are averaged.

\subsubsection{Water vapour}

The AOTF spectrometer had two detectors, collecting light from the two outputs of the AOTF with different polarizations. The main features of the spectrum are the spectral slope towards the longer wavelengths due to the solar spectrum, a large number of Fraunhofer lines, and some atmospheric absorption features, the most prominent being the $\mathrm{CO}_{2}$ absorption bands at $1.43,1.58$, and $1.6 \mu \mathrm{m}$, and the $\mathrm{H}_{2} \mathrm{O}$ absorption band around $1.38 \mu \mathrm{m}$ (Fig. 19). The latter band (Fig. 19) was used for the routine retrieval of the total column water vapour abundance in the atmosphere of Mars (Fedorova et al., 2006a). In the retrieval we generally use each individual spectrum in the sequence. An important issue is an accurate solar spectrum, because numerous solar lines frequently combine with the signatures of the Martian atmospheric gases. There are few sources of high-resolution solar spectral information in the spectral range of interest, and for the moment we are using the spectrum by Kurucz (1995) although its spectral resolution (sampled at $1 \mathrm{~cm}^{-1}$ ) is at the limit of what is required.

\subsubsection{Oxygen $\mathrm{O}_{2}\left({ }^{1} \Delta_{g}\right)$ emission}

On Mars, the emission of the dayglow $1.27 \mu \mathrm{m} \mathrm{O}_{2}\left({ }^{1} \Delta_{\mathrm{g}}\right)$ is produced when $\mathrm{O}_{3}$ is photo-dissociated by solar UV into $\mathrm{O}+\mathrm{O}_{2}$. The $\mathrm{O}_{2}$ molecule is produced in an excited state, which de-excites spontaneously while emitting one photon at $1.27 \mu \mathrm{m}$ (Fedorova et al., 2006b).

The band intensity observed by different authors from the ground varies from 1.5 to $26 \mathrm{MR}$ on Mars and, indeed, SPICAM IR routinely observes the $\mathrm{O}_{2}\left({ }^{1} \Delta_{\mathrm{g}}\right)$ band in Nadir and at the limb of Mars, mostly in the polar regions of the planet. Three spectra recorded along one orbit at different latitudes shown in Fig. 20 are different: the albedo is different, the shape of the spectrum, and the shape of $\mathrm{CO}_{2}$ absorption of spectrum 3 lines differ from 1 and 2 (see below), and finally, an emission feature near $1.27 \mu \mathrm{m}$ is apparent at spectra 2 and 3. This region zoomed at the right panel of Fig. 20 reveals a clear signature of $\mathrm{O}_{2}\left({ }^{1} \Delta_{\mathrm{g}}\right)$ band of the Martian atmosphere. On Venus, the same emission does exist, as said above, but it is present day and night, when $\mathrm{O}$ atoms produced by EUV photo-dissociation of $\mathrm{CO}_{2}$ recombine to form the $\mathrm{O}_{2}$ molecule. This is a major way to study the upper atmosphere circulation, much like the $\mathrm{NO}$ emission in the $\mathrm{UV}(\mathrm{N}+\mathrm{O}$ recombination $)$.

\subsubsection{Polarization}

Variations of polarization with wavelength and phase angle will be useful to determine some characteristics of 

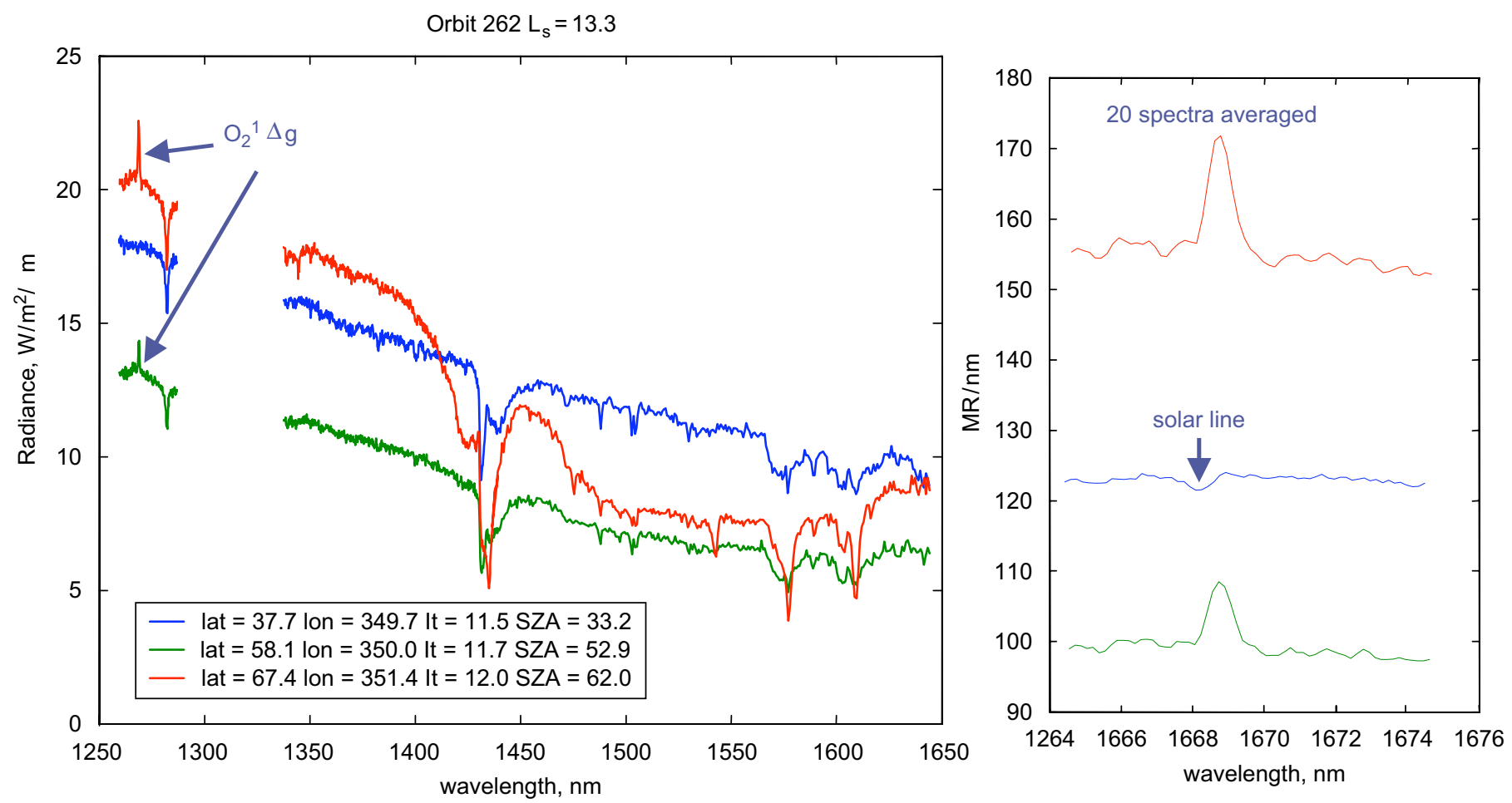

Fig. 20. Three spectra measured by SPICAM IR along the orbit 262 for latitudes of $38^{\circ}$ (blue -1 ), $58^{\circ}$ (green -2 ) and $67^{\circ}$ (red-3). On the right panel is enlarged the spectral region of $1.27 \mathrm{~mm} \mathrm{O}_{2}\left({ }^{1} \Delta_{\mathrm{g}}\right)$ emission band.

aerosols in the upper part of the clouds, and in the upper haze, if observed at the limb. However, because of the rather large FOV $\left(2^{\circ}\right)$, the spatial resolution at the limb will be coarser than desirable. We have measured the polarization power of the SPICAV IR spare flight model and indeed found that each channel is totally polarizing, and they are orthogonal.

\section{The SOIR spectrometer.}

An additional high-resolution IR solar occultation spectrometer $(2-4 \mu \mathrm{m})$ with a new optical concept was included in the SPICAV instrument, during the definition phase of VEX. It is a totally new instrument that will provide unique measurements from orbit with a resolving power of $15,000-20,000$, to measure $\mathrm{HDO} / \mathrm{H}_{2} \mathrm{O}$ and other isotopes and minor species at different altitudes.

\subsection{The scientific question of $\mathrm{HDO}$ and $\mathrm{H}_{2} \mathrm{O}$ in the upper atmosphere of Venus}

The present value of the $\mathrm{D} / \mathrm{H}$ ratio tells us something (but not everything) about the history of water on the planet. As mentioned earlier, deuterium in the lower atmosphere is found to be $\sim 150$ times more abundant on Venus than on Earth (Donahue et al., 1997; Donahue, 1999; De Bergh et al., 1991). This enrichment of $\mathrm{D} / \mathrm{H}$ is explained by preferential escape of $\mathrm{H}$ atoms from the upper atmosphere, with ion escape $\left(\mathrm{H}^{+}\right.$and $\left.\mathrm{D}^{+}\right)$being also important. The present water content and $\mathrm{D} / \mathrm{H}$ ratio can be interpreted either as the signature of an equivalent $3-\mathrm{km}$ a primordial ocean, lost at present (mainly by hydrodynamic escape), or a steady state in which water is continuously supplied to the surface by comets (Grinspoon and Lewis, 1988) or volcanism, or a non-steady regime combining the two sources. The present lifetime of the atmospheric water is highly uncertain but is likely less than $100 \mathrm{Myr}$ so that the primordial ocean is probably not the sole source of the present water. It may, however, be possible to derive constraints on the primordial water abundance by measuring precisely the atmospheric escape of water (i.e. of $\mathrm{H}$ atoms), and the fractionation factor describing the efficiency of $\mathrm{D}$ escape relative to $\mathrm{H}$ escape (Gurwell, 1995). The escape rate of $D$ and other atoms from the planetary exosphere depends, first, on their abundance in the upper atmosphere and, second, on the peculiarities of the interaction of the solar wind with the atmosphere. While escape of hydrogen is approximately known, the abundance of deuterium in the upper atmosphere of Venus, and furthermore its escape rate, is poorly constrained by optical observations (Bertaux and Clarke, 1989), though mass 2 ion measured by the ion mass spectrometer on Pioneer Venus is proposed to be $\mathrm{D}^{+}$, instead of $\mathrm{H}_{2}^{+}$, as was originally claimed (see the discussion above in the SPICAV UV section). The measurements of atom abundance and their vertical profile would be extremely important to quantify the escape processes. SPICAV UV might not be able to detect the $\mathrm{D}$ atoms $\mathrm{L} \alpha$ spike at the limb. However, the quantity of $\mathrm{D}^{+}$ions, of $\mathrm{D}$ atoms at escape level, and the $\mathrm{D}$ and $\mathrm{D}^{+}$escape rate, are certainly proportional to 
HDO abundance at the homopause level $(\sim 100 \mathrm{~km})$. Therefore, the measurement of HDO as high as possible will give quantitative indications of the present escape rate of $\mathrm{D}$ atoms.

An additional possible complication is the fact that HDO may undergo vertical fractionation (as on Earth). If there is a cold trap for HDO in the upper atmosphere of Venus, preventing all $\mathrm{D}$ escape, then possibly only $4.5 \mathrm{~m}$ of water ever existed on Venus, at most, and the mass 2 ion will be assigned to $\mathrm{H}_{2}^{+}$. This makes vertically resolved $\mathrm{HDO} / \mathrm{H}_{2} \mathrm{O}$ measurements very important to resolve this long-standing controversy about the nature of ion mass 2 . The same issue of $\mathrm{HDO} / \mathrm{H}_{2} \mathrm{O}$ fractionation is also of high significance on Mars (Bertaux and Montmessin, 2001).

SOIR will measure HDO profile up to $80-90 \mathrm{~km}$, therefore, characterizing escape of deuterium. Fig. 21 is a simulation of the atmospheric transmission of Venus for various tangent altitudes of the LOS. At $60 \mathrm{~km} \mathrm{SO}$ absorption contaminates HDO, but in this model we assumed an $\mathrm{SO}_{2}$ mixing ratio of $150 \mathrm{ppm}$ at this altitude, which is somewhat exaggerated because photolysis destroys $\mathrm{SO}_{2}$ above the clouds. Still one line of HDO can be detected (red arrow). Higher up, HDO is very well measurable at $60-70 \mathrm{~km}$, in the region where the "cold trap" may exist.

On Venus the dense atmosphere with opaque clouds located at $45-60 \mathrm{~km}$ prevents solar occultation sounding at low altitudes. High-resolution occultation studies are possible above the clouds, in the upper-cloud layer $(<90 \mathrm{~km})$ and higher. This allows unique measurements connected with the escape of water, and, therefore, to the evolution of water on the planet (see above). Another important scientific objective for SOIR is the measurement of the water vapour abundance profile up to $100 \mathrm{~km}$. In the range from 60 to $70 \mathrm{~km}$ this measurement can be performed in the band around $2560-2570 \mathrm{~cm}^{-1}(3.9 \mu \mathrm{m})$, which is expected to be unobstructed by any other absorbing constituent. The availability of the stronger 3760 $3860 \mathrm{~cm}^{-1}(2.56 \mu \mathrm{m})$ absorption band allows extending water vapour observations up to $100 \mathrm{~km}$. At an altitude of $60 \mathrm{~km}$ the $\mathrm{H}_{2} \mathrm{O}$ spectrum in this band shows few spectral lines unaffected by the absorption lines of $\mathrm{CO}_{2}$ and $\mathrm{HF}$, but at higher altitudes some isolated and useful $\mathrm{H}_{2} \mathrm{O}$ lines will become available. Other scientific targets of SOIR on Venus are: precision $\mathrm{H}_{2} \mathrm{O}$ profiling up to $\approx 105 \mathrm{~km}$; measurements of atmospheric density up to very high altitudes, though complicated due to non-LTE effects; measurements of $\mathrm{CO}_{2}$ and $\mathrm{H}_{2} \mathrm{O}$ isotopes (besides $\mathrm{HDO}$ ); measurements of known minor constituents $(\mathrm{CO}, \mathrm{HF}, \mathrm{HCl}$, $\mathrm{SO}_{2}, \mathrm{H}_{2} \mathrm{~S}$, OCS); sensitive search for new minor species $\left(\mathrm{C}_{2} \mathrm{H}_{2}, \mathrm{CH}_{4}, \mathrm{NO}, \mathrm{N}_{2} \mathrm{O}, \mathrm{H}_{2} \mathrm{CO}, \mathrm{HCN}\right.$ and many others). Further simulations of the atmospheric transmission in the SOIR total bandwidth are shown on Fig. 22.

\subsection{Technical description of SOIR spectrometer channel}

A full description of this novel instrument may be found in Nevejans et al. (2006). SOIR is mounted on top of the first floor containing SPICAV UV and IR channels. The main idea of SOIR optical concept consists of using a grating that gives a higher resolution and dispersion than ordinary gratings, i.e. an echelle grating, known to yield high reciprocal dispersion and high throughput in a compact design. Since the echelle has overlapping orders, some order sorting filtering is required. In this design the order sorting is established by means of an AOTF, which has the advantage of not needing any mechanical moving mechanisms (at variance with the classical solution of a filter wheel). The usage of an AOTF introduces the possibility of a quick random access to any grating order by electronic command. Also, as in SPICAV NIR, the deactivation of the AOTF allows to stop any solar input to the spectrometer, thus measuring a thermal background spectrum that can be subtracted from the observed

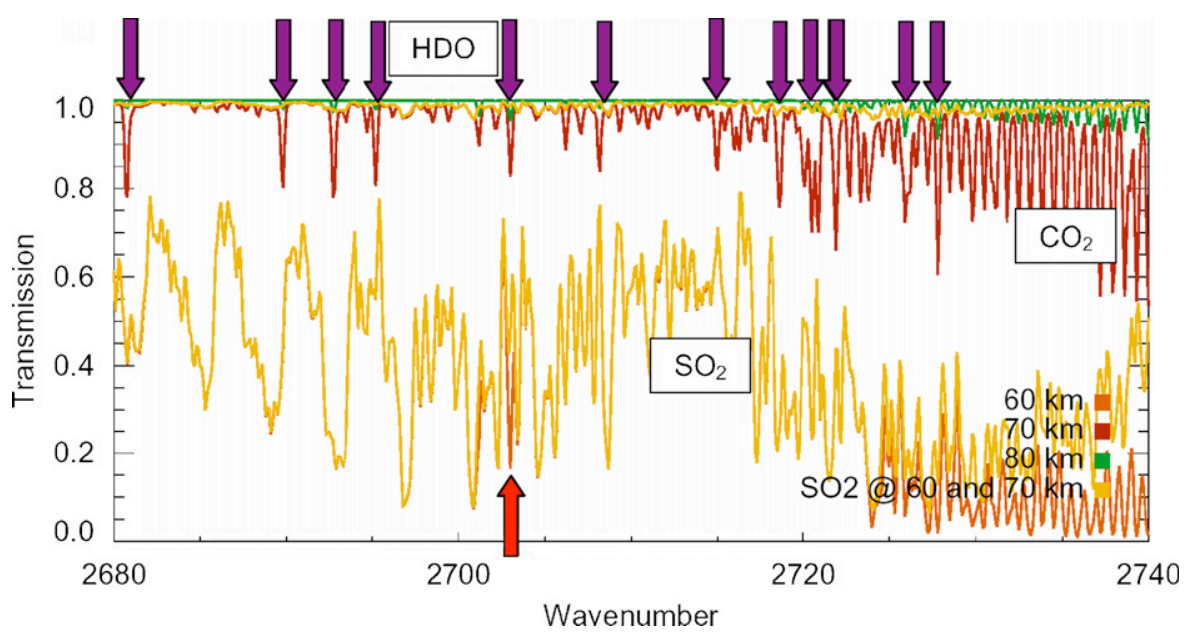

Fig. 21. Simulation of the atmospheric transmission of Venus for various tangent altitudes of the LOS. At $60 \mathrm{~km} \mathrm{SO}_{2}$ absorption contaminates HDO, but in this model the quantity of $\mathrm{SO}_{2}$ is somewhat exaggerated. Still one line of $\mathrm{HDO}$ can be detected (red arrow) at $60 \mathrm{~km}$. HDO is very well measurable at $60-70 \mathrm{~km}$, in the region where the "cold trap" may exist. In this simulation a mixing ratio of $5 \mathrm{ppm}$ was assumed for $\mathrm{H}_{2} \mathrm{O}$, and $\mathrm{HDO} / \mathrm{H}_{2} \mathrm{O}=120$ times terrestrial. 

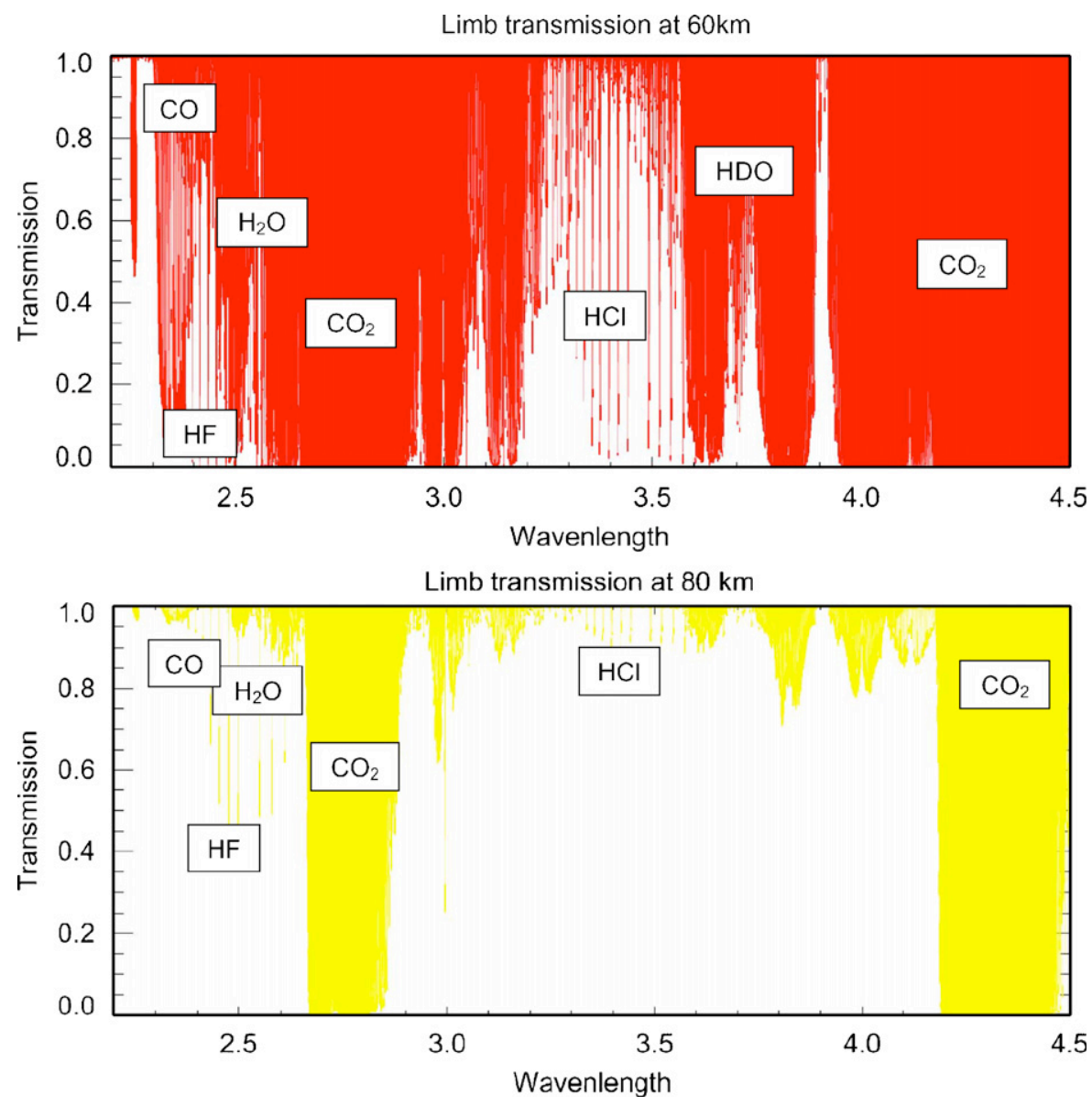

Fig. 22. Computed limb transmission of the Venus atmosphere at grazing altitudes of 60 and $80 \mathrm{~km}$, in the wavelength domain of SOIR. Various gases are absorbing in different wavelength regions.

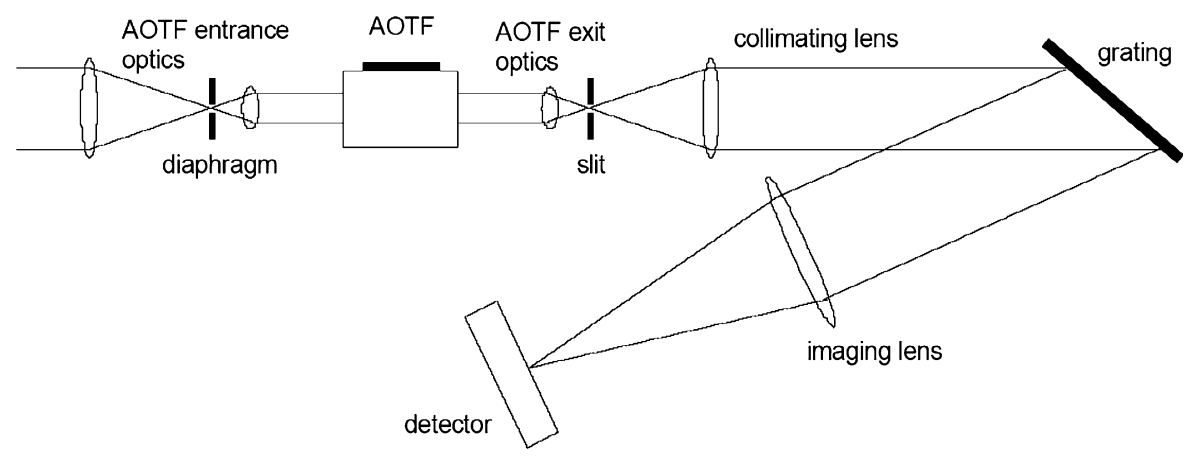

Fig. 23. Simplified optical scheme of SOIR. See text for detailed explanations.

spectrum (no need of a mechanical chopper). Fig. 23 represents the simplified set-up of the compact highresolution spectrometer using an AOTF-echelle combination. The optical scheme was designed in detail by OIP Sensor Systems in Belgium; the electronics were designed by BIRA-IASB. The spectrometer is composed of three main parts: (a) the front-end part which collects the solar light, defines the FOV and selects the observed wavelength domain; (b) the spectrometer itself, which realizes the desired spectral sampling interval and the spectral resolu- tion and finally, (c) the detector system, which records the spectra.

The front-end starts at the AOTF entrance optics that reduces the diameter of the incoming light beam so that it becomes compatible with the size of the acceptance aperture of the AOTF. In order to have the solar light entering the instrument along its optical axis, a two-mirror periscope is mounted externally at the entrance of the spectrometer. In the entrance optics intermediate image plane a first diaphragm limits the FOV to slightly larger 
than the desired FOV, in order to block unwanted light and to avoid light scattering and ghost images. Then the beam enters the AOTF, which is used as the ordersorting filter of the system. When AOTF is ON, it directs along its optical axis a small spectral fraction of incidence beam, aligned to the free spectral range (FSR) of the spectrometer: the range of wavelengths in which there is no interference or superposition of light from adjacent orders. The AOTF exit optics images the beam on the spectrometer entrance slit. The spectrometer slit is actually the entrance aperture for the spectrometer part of the instrument. A collimating lens captures the light passing through the spectrometer entrance slit and forms a parallel beam to the echelle grating, used near the Littrow configuration. Finally, the light diffracted by the grating is imaged via a camera lens on the detector. With 4 grooves per $\mathrm{mm}$ the $\arctan (2)$ incidence-grating SOIR spectrometer operates on diffraction orders from 101 (corresponding to the wavelengths of $\sim 4.4 \mu \mathrm{m}$ ) to 194 $(\sim 2.3 \mu \mathrm{m})$.

The principal components of the instrument are:

- Custom AOTF from AFAR, Russia (Table 4).

- Custom echelle grating from Bach Research, Boulder, USA (Table 3).

- Customized 2D MCT detector from SOFRADIR, France (Table 5).

- SOIR instrument is controlled by a dedicated space qualified FPGA-based electronics unit, designed at BIRA-IASB.

Table 4

\section{Material}

Wavelength range

Spectral bandwidth (FWHM)

Acceptance aperture in plane of diffraction

Acceptance aperture perpendicular to plane of diffraction

Acceptance angle in plane of diffraction

Acceptance angle perpendicular to plane of diffraction

Diffraction angle

RF frequency range

$\mathrm{TeO}_{2}$
2.325 to $4.250 \mu \mathrm{m}$
$<22 \mathrm{~cm}^{-1}$
$>10 \mathrm{~mm}$
$>4 \mathrm{~mm}$
$\pm 2^{\circ}$
$\pm 3^{\circ}$
$4.6^{\circ}$
$13.5-25 \mathrm{MHz}$

Table 5

\section{Wavelength range}

Diffraction orders

Steep edge angle $=$ blaze angle

Number of grooves per $\mathrm{mm}$

Groove spacing

Total number of grooves $(\mathrm{N})$

Ruled area

Blank dimensions

Blank material

Coating material
Our customized version is derived from the standard Sofradir model ID MM0067. It contains an IR optoelectronic device sensitive to radiation in the $1.7-4.3 \mu \mathrm{m}$ spectral region. However, a window filters between 2.2 and $4.3 \mu \mathrm{m}$. The device includes a high-sensitivity focal plane array (FPA) comprized of a two-dimensional detector array made of photovoltaic Mercury Cadmium Telluride ( $\mathrm{HgCdTe} / \mathrm{MCT})$ pixels organized as 320 columns by 256 rows, encapsulated in vacuum. This permits operating in air, with full cooling and without risk of $\mathrm{H}_{2} \mathrm{O}$ condensation: an important feature that simplifies developing and testing the instrument. The sensitive area of $9600 \mu \mathrm{m} \times 7680 \mu \mathrm{m}$ has a fill factor greater than $90 \%$ and is composed of pixels of $30 \mu \mathrm{m} \times 30 \mu \mathrm{m}$. All pixels are connected by means of indium bumps to a CMOS Readout Integrated Circuit (ROIC) that forms a sandwich with the MCT array. The ROIC reads the charge from each MCT photovoltaic diode via direct injection and integrates it on the pixel input capacitor (selectable 0.7 or $2.1 \mathrm{pF}$ ) during a programmable integration time. Then the charges are converted to voltages, which appear on the serial outputs multiplexed per row and then per column. Although the ROIC allows for different ways and speeds of detector read-out, for power, telemetry and simplicity reasons the detector read-out is limited to a programmable window of 8 lines of 320 pixels, selectable within the detector area, through a single output at a rate of 1 megapixel/s. The encapsulated detector is cooled by a Ricor mechanical cryo-cooling machine using a Stirling cycle, as a part of the Sofradir integrated detector, that allows reaching a detector temperature of $90 \mathrm{~K}$ within a few minutes. The

Table 6

\begin{tabular}{|c|c|c|}
\hline \multirow{4}{*}{$\begin{array}{l}2.325 \text { to } 4.250 \mu \mathrm{m} \\
<22 \mathrm{~cm}^{-1} \\
>10 \mathrm{~mm} \\
>4 \mathrm{~mm}\end{array}$} & \multirow[b]{2}{*}{ IR detector material } & \multirow[b]{2}{*}{$\begin{array}{l}\text { Mercury Cadmium Telluride } \\
(\mathrm{HgCdTe})\end{array}$} \\
\hline & & \\
\hline & Covered IR wavelength range & 1.66 to $4.4 \mu \mathrm{m}(\mathrm{FWHM})$ \\
\hline & Operating temperature & $<110 \mathrm{~K}$ \\
\hline & Pixel configuration & 2-D array organized as 320 columns by \\
\hline $\pm 2^{\circ}$ & & 256 rows \\
\hline $\pm 3^{\circ}$ & & Programmable windowing \\
\hline \multirow{5}{*}{$\begin{array}{l}4.6^{\circ} \\
13.5-25 \mathrm{MHz}\end{array}$} & Pixel size & $30 \mu \mathrm{m} \times 30 \mu \mathrm{m}$ \\
\hline & Output level & 1.6 (no light) to $4.4 \mathrm{~V}$ (full well) \\
\hline & Linearity (typical) & $\pm 1 \%$ between 25 and $92 \%$ of full well \\
\hline & Gain setting & $\begin{array}{l} \pm 2 \% \text { between } 10 \text { and } 98 \% \text { of full well } \\
0.7 \text { or } 2.1 \mathrm{pF} \text { integration capacitor }\end{array}$ \\
\hline & $\begin{array}{l}\text { Responsitivity (measured over } \\
\text { complete wavelength range) }\end{array}$ & $\begin{array}{l}\text { Typical } 76 \mathrm{~V} / \mathrm{nW} \text { per pixel @ } 20 \mathrm{~ms} \\
\text { integration time and gain } \\
\text { setting }=0.7 \mathrm{pF}\end{array}$ \\
\hline 2.2 to $4.3 \mu \mathrm{m}$ & Read-out speed & 1 to $6 \mathrm{MHz}$ for single output \\
\hline 100 to 200 & Integration time & Minimum $3 \mu \mathrm{s}$ \\
\hline $63.43^{\circ}=\arctan (2)$ & & Acceptance tested at $20 \mathrm{~ms}$ \\
\hline 4 & Detector mounting & Mounted in dewar with $\mathrm{f} / 4$ cold screen \\
\hline $\begin{array}{l}250 \mu \mathrm{m} \\
568\end{array}$ & Integrated microcooler model & $\begin{array}{l}\text { K508, modified for space applications } \\
\text { (RICOR, Israel) }\end{array}$ \\
\hline $142 \times 56 \mathrm{~mm}^{2}$ & Steady state cooling power & $4.1 \mathrm{~W}$ typical at $24 \mathrm{~V}$ supply voltage (in \\
\hline $150 \times 60 \times 25 \mathrm{~mm}^{3}$ & (begin of life) & $20^{\circ} \mathrm{C}$ air) \\
\hline Aluminium 6061-T6 & Pre-cooling power (begin of & $9.4 \mathrm{~W}$ typical at $24 \mathrm{~V}$ supply voltage (in \\
\hline $\mathrm{Au}$ & life) & $20^{\circ} \mathrm{C}$ air $)$ \\
\hline
\end{tabular}


heat of SOIR is conducted to a side panel of VEX that is not illuminated by the Sun in normal conditions. The main characteristics of the customized version of the detector are summarized in Table 6 .

SOIR has undergone numerous tests on ground, the most elaborate using the Sun as the source of light. The solar rays passing the Earth's atmosphere were reflected by a mirror outside the lab and directed along the optical axis of SOIR, which was located in a clean room inside. Spectra corresponding to all orders of the echelle grating between 2.2 and $4.3 \mu \mathrm{m}$ were recorded. A typical read-out, recorded spectrometer order 133, and showing the spectral features of atmospheric methane and $\mathrm{H}_{2} \mathrm{O}$ is shown in Fig. 24 and compared to a synthetic spectrum of the atmospheric transmission and solar spectrum. The water vapour absorption dominates, but the methane line at $2978 \mathrm{~cm}^{-1}$ is clearly seen, in a region where water vapour is not saturated. Such an instrument around Mars would certainly be useful to understand the puzzling problem of methane. In view of $\mathrm{S} / \mathrm{N}$ calculations and actual laboratory performance, it is clear that SOIR should be able to measure a $1 \%$ absorption feature when the solar flux is not too much attenuated by the haze continuous absorption.

\subsection{SOIR and nightside, dayside, and solar observations}

When a solar occultation will be programmed, VEX will be oriented with the solar entry ports of all three spectrometers of SPICAV to the Sun. Solar spectra will be recorded by SOIR, first above the atmosphere, then through the atmosphere. Then, with the orientation of VEX fixed, the LOS of SOIR will see the nightside of Venus. The question arises then if SOIR will see the thermal emission coming from the ground and lower atmosphere, which is very rich in information, since it crosses all altitudes. In fact, the difference of brightness at $3 \mu \mathrm{m}$ between the disc of the Sun and Venus ground at $700 \mathrm{~K}$ is only a factor of 210 . When looking at the Sun from the lab, the detector was saturated in $10 \mathrm{~ms}$. In order to gain sensitivity, we can program SOIR with a $5 \mathrm{~s}$ integration time, and we can sum a large number of lines of the detector (numerically). The actual signal will depend crucially on the transparency of the cloud deck to radiation at $3 \mu \mathrm{m}$, which seems to be highly variable. If it works, it would open a very important way to study the lower atmosphere of Venus. Indeed, the spectral resolution of SOIR is $5-10$ times the resolution of PFS, allowing the detection of HDO in the lower atmosphere (as was done
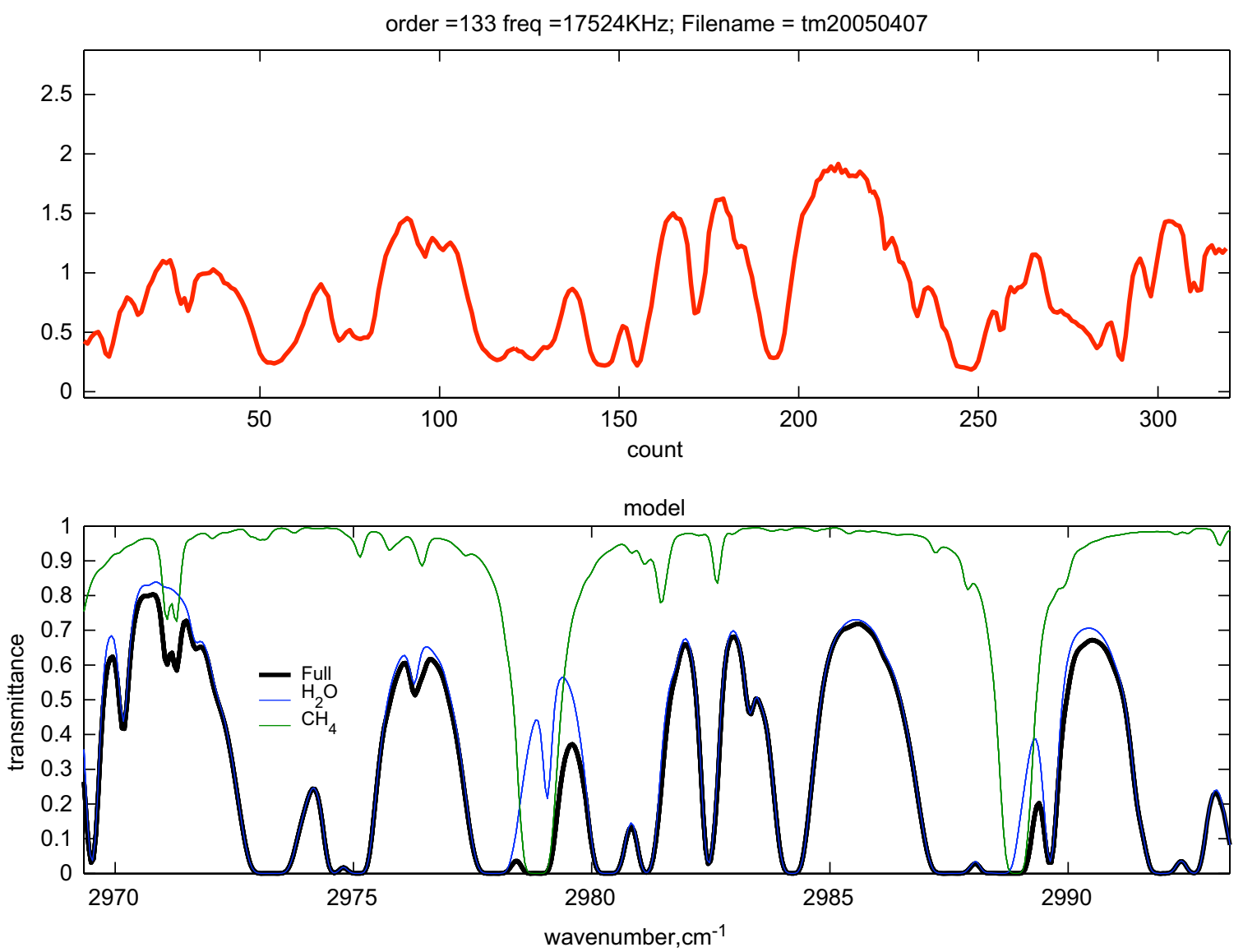

Fig. 24. Comparison of Earth atmosphere transmission model with SOIR data for order 133. Top: red curve- spectrum obtained by applying 4-to-1 binning on 32 detector lines of 320 pixel each. Bottom: black - model of Earth atmosphere, green $-\mathrm{CH}_{4}$ only, blue $-\mathrm{H}_{2} \mathrm{O}$ only. Adopted resolving power for the synthetic spectra is 20,000 . 


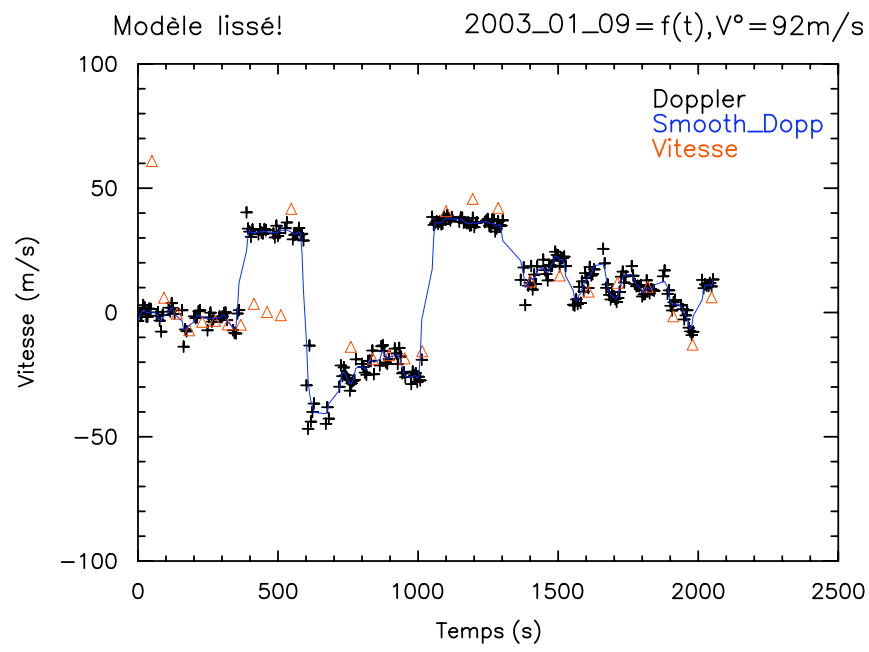

Fig. 25. Doppler shift measured in the reflected solar light on various points of the disc of Venus (red triangles) at Observatoire de Haute Provence with Emilie spectrometer and Astronomical Absolute Accelerometer (Gabsi et al., 2006). Black + : forward model assuming a solid rotation of the upper atmosphere at an equatorial velocity of $92 \mathrm{~m} / \mathrm{s}$.

from Earth), $\mathrm{H}_{2} \mathrm{O}$ and possibly unforeseen minor constituents. Dayside measurements will also be attempted. We will mainly explore the spectral regions which escaped the measurements of Connes et al. (1979) because of terrestrial absorption, at a spectral resolution which has never been achieved in space in this wavelength region. Even the solar spectrum recorded before the occultations will be a reference in the future. A full solar spectrum has been already acquired during the cruise to Venus.

\section{Ground-based supporting campaigns for wind measurements}

Venus winds will be measured by VMC, on the dayside by looking at the displacement of blue-UV markings. There is, however, the lingering problem of knowing precisely what is measured. Like on Earth, there may be waves of condensation that contribute to the appearance of the markings, and the measured quantity is not really a material displacement, but a phase motion. Indeed, some UV markings are actually locked in local time (around the sub-solar point), which proves this.

One of us (J.L. Bertaux) has designed a method to measure the material displacement of the cloud particles, by recording the spectrum of the solar light scattered by the particles, and measuring the Doppler shift. It has been already proven with the Emilie spectrometer at Observatoire de Haute Provence; this spectrometer is equipped with a high-precision calibration system, the Absolute Astronomical Accelerometer (invented by Pierre Connes). Winds of the order of $60-90 \mathrm{~m} / \mathrm{s}$ have been recorded (Fig. 25), which is totally in line with the 4 days rotation (Gabsi et al., 2006). Therefore, it is suggested to organize coordinated campaigns, in which there would be simultaneous observations from VEX and from the ground, to disen- tangle material motions of the particles, from the wave phase motion.

\section{Acknowledgements}

Venus Express is a space mission from ESA (European Space Agency). We wish to express our gratitude to all ESA members who participated to the preparation of this mission developed in an extremely short time: Hakan Svedhem as Project Scientist, Hans Eggel for his constant technical dedication, Don McCoy as Project Manager, Jacques Louet as Technical Director of Space Science Directorate at ESTEC, and Raymond Hoofs for the development of operation software. We acknowledge the important role of Dima Titov, trying to put together contradictory requirements from the various PIs. We thank also EADS/Astrium Corp. for the design and construction of the VEX spacecraft, and in particular Alain Clochet, responsible for the payload. We thank our collaborators at the three institutes for the design and fabrication of the instrument (Service d'Aéronomie/France, BIRA/Belgium and IKI/Moscow), including the important help of JeanLuc Maria and Mustapha Meftah at Service d'Aéronomie during emergency crisis. We wish to thank CNRS and CNES for financing SPICAM in France, the Russian Academy of Sciences for support at IKI and NASA for support of US co-investigators. We wish to express our gratitude for the financial support we received from the Belgian Federal Science Policy Office, who completely funded the design and manufacturing of SOIR as well part of the construction of SPICAM on Mars Express (ESA PRODEX contract nr. 90113) during years 2003-2005.We wish to thank Francis Rocard at CNES, Jean-Claude Lalaurie at Centre Spatial de Toulouse (CNES) for his support and help in some difficult circumstances. Finally, we wish to acknowledge the excellent work of the Sofradir company, which developed a custom design mechanically cooled detector of very high performance in a relatively short time and totally on schedule, and we wish to thank particularly Sofradir Chairman Executive Officer, Mr. Bensousan.

\section{References}

Acuña, M.H., et al., 2001. Magnetic field of Mars: summary of results from the aerobraking and mapping orbits. J. Geophys. Res. 106, 23,403-23,417.

Allen, D.A., Crawford, J.W., 1984. Cloud structure on the dark side of Venus. Nature 307, 222-224.

Baines, K.V., et al., 2000. Detection of sub-micron radiation from the surface of Venus by Cassini VIMS. Icarus 148, 307-311.

Barth, C.A., Pearce, J.B., Kelly, K.K., Wallace, L., Fastie, W.G., 1968. Science 158, 1675.

Barth, C.A., Hord, C.W., Pearce, J.B., Kelly, K.K., Anderson, G.P., Stewart, A.I., 1971. Mariner 6 and 7 ultraviolet spectrometer experiment: Upper atmosphere data. J. Geophys. Res. 76, 2213-2227.

Barth, C.A., Stewart, A.I.F., Bougher, S.W., Hunten, D.M., Bauer, S.J., Nagy, A.F., 1992. In: Mars, K., et al. (Eds.), Aeronomy of the current Martian atmosphere, University of Arizona Press. 
Bertaux, J.L., Clarke, J.T., 1989. Deuterium content of the Venus atmosphere. Nature 338, 567-568.

Bertaux, J.L., Montmessin, F., 2001. Isotopic fractionation through water vapor condensation: the Deuteropause, a cold trap for Deuterium in the atmosphere of Mars. JGR Planets 106, E 12, 32879-32884.

Bertaux, J.L., Blamont, J.E., Marcelin, M., Kurt, V.G., Smirnov, A.S., 1979. Consequences of the day-to-night variation of theVenus exospheric temperature. Nature 277, 546-548.

Bertaux, J.L., Blamont, J.E., Lépine, V.M., Kurt, V.G., Romanova, N.N., Smirnov, A.S., 1981. Venera 11 and Venera 12 observations of E.U.V. emissions from the upper atmosphere of Venus. Planet Space Sci. 29, 149-166.

Bertaux, J.L., Lépine, V.M., Kurt, V.G., Smirnov, A.S., 1982. Altitude profile of $\mathrm{H}$ in the atmosphere of Venus from Lyman-alpha observations of Venera 11 and Venera 12 and origin of the hot exospheric component. Icarus 52, 221-224.

Bertaux, J.L., Widemann, T., Hauchecorne, A., Moroz, V.I., Ekonomov, A.P., 1996. Vega-1 and Vega-2 entry probes: an investigation of local UV absorption $(220-400 \mathrm{~nm})$ in the atmosphere of Venus $\left(\mathrm{SO}_{2}\right.$, aerosols, Cloud Structure). J. Geophys. Res. 101, 12,709-12,745.

Bertaux, J.L., Fonteyn, D., Korablev, O., Chassefière, E., Dimarellis, E., Dubois, J.P., Hauchecorne, A., Cabane, M., Ranou, P., LevasseurRegourd, A.C., Cernogora, G., Quemerais, E., Hermans, C., Kockarts, G., Lippens, C., De Maziere, M., Moreau, D., Muller, C., Neefs, E., Simon, P.C., Forget, F., Hourdin, F., Talagrand, O., Moroz, V.I., Rodin, A., Sandel, B., Stern, A., 2000. The study of the Martian atmosphere from top to bottom with SPICAM Light on Mars Express. Planet. Space Sci. 48, 1303-1320.

Bertaux, J.L., Leblanc, F., Perrier, S., Quémerais, E., Korablev, O., Dimarellis, E., Reberac, A., Forget, F., Simon, P.C., Stern, A.S., Sandel, B.R., and the SPICAM team, 2005a. First observation of nightglow in the upper atmosphere of Mars: the NO bands in UV and implications for atmospheric transport. Science 307, 566.

Bertaux, J.L., Leblanc, F., Witasse, O., Quémerais, E., Lilensten, J., Stern, S.A., Sandel, B., Korablev, O., 2005b. Discovery of aurora on Mars. Nature 435 (7043), 790-794.

Bertaux, J.L., Korablev, O., Perrier, S., Quémerais, E., Montmessin, F., Leblanc, F., Lebonnois, S., Lefèvre, F., Forget, F., Fedorova, A., Rannou, P., Dimarellis, E., Reberac, A., Fonteyn, D., Chaufray, J.Y., Guibert, S., and the SPICAM Team, 2006. SPICAM on Mars Express: observing modes and overview of UV Spectrometer data and scientific Results. J. Geophys. Res., in press.

Bougher, S.W., Gerard, J.C., Stewart, A.I.F., Fesen, C.G., 1990. J. Geophys. Res. 95, 6271-6284.

Bougher, S.W., Roble, R.G., Ridley, E.C., Dickinson, R.E., 1990. The Mars thermosphere. II. General circulation with coupled dynamics and composition. J. Geophys. Res. 95, 14811-14827.

Bougher, S.W., Alexander, M.J., Mayr, H.G., 1997. In: Bougher, S.W., Hunten, D.M., Phillips, R.J. (Eds.), Venus II. The University of Arizona Press, p. 1997.

Boyer, C., Camichel, H., 1961. Observations photographiques de la planète Vénus. Ann. Astrophys. 24, 531-535.

Boyer, C., Camichel, H., 1965. Étude photographique de la rotation de Vénus. C.R. Acad. Sci., Paris 260, 809-810.

Cheng, B.M., Chew, E.P., Liu, C.-P., Bahou, M., Lee, Y.-P., Yung, Y.L., Gerstell, M.F., 1999. Photo-induced fractionation of water isotopomers in the Martian atmosphere. Geophys. Res. Lett. 26, 3657-3660.

De Bergh, C., Bezard, B., Owen, T., Crisp, D., Maillard, J.-P., Lutz, B.L., 1991. Deuterium on Venus: observations from Earth. Science 251, 547-549.

Connes, P., Noxon, J.F., Traub, W.A., Ca rleton, N.P., 1979. O $2(1 \Delta)$ emission in the day and night airglow of Venus. Astrophys. J. 233, L29-L32.

Donahue, T.M., 1999. New Analysis of Hydrogen and Deuterium Escape from Venus. Icarus 141, 226-235.

Donahue, T.M., Hoffman, J.H., Hodges, R.R., Watson, A.J., 1982. Venus was wet - a measurement of the ratio of deuterium to hydrogen. Science 216, 630-633.
Donahue, T.M., Grinspoon, D.H., Hartle, R.E., Hodges, R.R., 1997. In: Bougher, S.W., Hunten, D.M., Philipps, R.J. (Eds.), Ion/Neutral Escape of Hydrogen and Deuterium: evolution of water, Chapter in book Venus II, University of Arizona, pp. 385-414.

Esposito, L.W., Bertaux, J.L., Krasnopolsky, V., Moroz, V.I., Zasova, L.L., 1997. In: Bougher, S.W., Hunten, D.M., Philipps, R.J. (Eds.), Chemistry of Lower Atmosphere and Clouds, Chapter in book Venus II, University of Arizona, pp. 415-458.

Esposito, L.W., 1984. Sulfur dioxide: episodic injection shows evidence for active Venus volcanism. Science 223, 1072-1074.

Fegley, B., Zolotov, M.Y., Lodders, K., 1997. The oxidation state of the lower atmosphere and surface of Venus. Icarus 125 (2), 416-439.

Feldman, P.D., Moos, H.W., Clarke, J.T., Lane, A.L., 1979. Nature 279, 221.

Feldman, P.D., Burgh, E.B., Durrance, S.T., Davidsen, A.F., 2000. Farultraviolet spectroscopy of Venus and Mars at $4 \AA$ resolution with the Hopkins Ultraviolet Telescope on Astro-2. Astrophys. J. 538, 395.

Fedorova, A., Korablev, O., Bertaux, J.L., Rodin, A., Perrier, S., Kiselev, A., 2006a. Mars water vapor abundance from AOTF IR SPICAM spectrometer: seasonal and geographic distribution, J. Geophys. Rev., in press.

Fedorova, A., Korablev, O., Perrier, S., Bertaux, J.L., Lefevre, F., Rodin, A., 2006b. Observation of $\mathrm{O}_{2} 1.27 \mu \mathrm{m}$ dayglow by SPICAM IR: seasonal distribution for first Martian year of Mars-Express, J. Geophys. Rev., in press.

Forget, F., Lebonnois, S., Angelats, M., Coll, I., Quémerais, E., Bertaux, J.L., Montmessin, F., Dimarellis, E., Reberac, A., Lopez-Valverde, M., Gonzalez Galindo, F., 2006. Mars atmosphere density and temperature between 50 and $130 \mathrm{~km}$ observed by Mars Express SPICAM stellar occultation, Second International Workshop on Mars Atmosphere modelling and observations, March 2006, Granada, Spain.

Fox, J.L., 1986. Models for aurora and airglow emissions from other planetary atmospheres. Can. J. Phys. 64, 1631-1656.

Gabsi, Y., Bertaux, J.L., Schmitt, J., Guibert, S., Hauchecorne, A., 2006. Measurements of wind velocity on Venus with Absolute Accelerometry, in preparation.

Glenar, D.A., Hillman, J.J., Saiff, B., Bergstralh, J., 1994. Acouto-optic imaging spectropolarimery for remote sensing. Appl. Opt. 33 (31), 7412-7424

Grinspoon, D.H., Lewis, J.S., 1988. Cometary water on Venusimplications of stochastic impacts. Icarus 74, 21-35.

Gurwell, M.A., 1995. Evolution of deuterium on Venus. Nature 378, $22-23$.

Hord, C.W., et al., 1991. Science 253, 1548.

Korablev, O.I., Bertaux, J.-L., Dubois, J.-P., 2001. Occultation of stars in the UV: study of the atmosphere of Mars. J. Geophys. Res. 106 (E4), 7597-7610.

Korablev, O.I., Bertaux, J.-L., Vinogradov, I.I., 2002a. Compact high-resolution IR spectrometer for atmospheric studies. Proc. SPIE 4818.

Korablev, O., Bertaux, J.-L., Grigoriev, A., Dimarellis, E., Kalinnikov, Yu., Rodin, A., Muller, C., Fonteyn, D., 2002b. An AOTF-based spectrometer for the studies of Mars atmosphere for Mars Express ESA mission. Adv. Space Res. 29 (2), 143-150.

Korablev, O.I., Bertaux, J.L., Dimarellis, E., Grigoriev, A., Kalinnikov, Yu., Stepanov, A., Guibert, S., 2002c. AOTF-based spectrometer for Mars atmosphere sounding. Proc. SPIE 4818, 261-271.

Korablev, O.I., Bertaux, J.-L., Vinogradov, I.I., Kalinnikov, Yuk, Nevejans, D., Neefs, E., 2004. Compact high-resolution echelle-AOTF NIR spectrometer to study the details of planetary atmospheres. ESA SP 554, 73-80.

Korablev, O., Bertaux, J.L., Fedorova, A., Perrier, S., Stepanov, A., Kalinnikov, Yu., Kiselev, A., Grigoriev, A., Jegoulev, V., Dimarellis, E., Dubois, J.P., Reberac, A., Van Ransbeeck, E., Gondet, B., Montmessin, F., Rodin, A., 2006. Spicam IR acousto-optic spectrometer experiment on Mars Express, J. Geophys. Rev., submitted for publication. 
Krasnopolsky, V.A., Tomashova, G.V., 1980. Venus night airglow variations. Cosmic Res. 18, 766.

Krasnopolsky, V.A., Mumma, M.J., Gladstone, G.R., 1998. Detection of atomic deuterium in the upper atmosphere of Mars. Science 280, $1576-1580$

Kurucz, R., 1995. The solar spectrum: atlases and line identifications. In: Saival, A.J., Blomme, R., Grevesse, N. (Eds.), Workshop on Laboratory and Astronomical High resolution Spectra, Astronomical society of the Pacific Conference Series, Proceeding of ASP conference, vol.81, held in Brussels, Belgium, 29 August-2 September 1994, San Francisco, Astronomical Society of the Pacific (ASP), p. 17

Leblanc, F., Chaufray, J.Y., Lilensten, J., Witasse, O., Bertaux, J.L., and the SPICAM Team, 2006. The Martian dayglow as seen by SPICAM UV spectrometer on Mars Express, J. Geophys. Rev., in press.

Lebonnois, S., Quémerais, E., Montmessin, F., Lefèvre, F., Perrier, S., Bertaux, J.L., Forget, F., 2006. Vertical distribution of ozone on Mars as measured by SPICAM/Mars-Express using stellar occultations. J. Geophys. Rev., in press.

McElroy, M.B., Prather, M.J., Rodriguez, J.M., 1982. Escape of hydrogen from Venus. Science 215, 1614-1615.

Montmessin, F., Quémerais, E., Bertaux, J.L., Korablev, O., Fedorova, A., Rannou, P., Lebonnois, S., 2006. Stellar Occultations at UV wavelengths by the SPICAM instrument: retrieval and analysis of Martian haze profiles, J. Geophys. Rev., in press.

Nagy, A.F., Cravens, T.E., 1988. Hot oxygen in the upper atmospheres of Venus an Mars. Geophys. Res. Lett. 8, 629-632.

Nevejans, D., Neefs, E., Van Ransbeeck, E., Berkenbosch, S., Clairquin, R., De Vos, L., Moelans, W., Glorieux, S., Baeke, A., Korablev, O., Vinogradov, I., Bach, B., Dubois, J.P., Villard, E., 2006. Compact high-resolution space-borne echelle grating spectrometer with AOTF based order sorting for the infrared domain from 2.2 to 4.3 micrometer, Appl. Opt., submitted for publication.
Paxton and Anderson, 1992. In: Luhmann, J.G., Tatrallyay, M., Pepin, R.O. (Eds.), Venus and Mars: Atmospheres, Ionospheres, and Solar Wind Interactions, (Geophys. Monogr. 66; Washington, DC: AGU), p. 191.

Perrier, S., Bertaux, J.L., Lefèvre, F., Lebonnois, S., Korablev, O., Fedorova, A., Montmessin, F., 2006. Global climatology of ozone on Mars from SPICAM/MEX UV measurements. J. Geophys. Rev., in press.

Philips, J.L., Stewart, A.I.F., Luhmann, J.G., 1986. The Venus ultraviolet aurora: observations at $130.4 \mathrm{~nm}$. Geophys. Res. Lett. 13, 1047-1050.

Quémerais, E., Bertaux, J.L., Korablev, O., Dimarellis, E., Cot, C., Sandel, B. R., Fussen, D., 2006. Stellar Occultations observed by SPICAM on Mars Express, J. Geophys. Rev., in press.

Rannou, P., Bertaux, J.L., Perrier, S., Montmessin, F., Reberac, A., 2006. Dust and cloud detection at Mars Limb from UV scattered sunlight with SPICAM, J. Geophys. Rev., submitted for publication.

Roscoe, H.K., Freshwater, R.A., Wolfenden, R., Jones, R.L., Fish, D.J., Harries, J.E., Oldham, D.J., 1994. Using stars for remote sensing of the Earth's stratosphere. Appl. Optics. 33, 7126-7131.

Santer, R., Deschamps, M., Ksanfomaliti, L.V., Dollfus, A., 1985. A Photopolarimetric analysis of the Martian atmosphere by the Soviet MARS-5 orbiter. I-White clouds and dust veils. Astron. Astrophys. 150 (2), 217-228.

Smith, G.R., Hunten, D.M., 1990. Study of planetary atmospheres by absorptive occultations. Rev. Geophys. 28, 117.

Stewart, A.I., Barth, C.A., Hord, C.W., 1972. Mariner 9 Ultraviolet spectrometer experiment: structure of Mars' upper atmosphere. Icarus 17, 469-474.

Stewart, A.I.F., Anderson Jr., D.E., Esposito, L.W., Barth, C.A., 1979. Science 203, 777.

Stewart, A.I.F., et al., 1980. J. Geophys. Res. 85, 7861-7870.

Stewart, A.I.F., Barth, C.A., 1979. Science 205, 59.

Trauger, J.T., Lunine, J.I., 1983. Spectroscopy of molecular oxygen in the atmospheres of Venus and Mars. Icarus 55, 272. 